\title{
BREAST CANCER TOMOTHERAPY: THE IMPACT OF INTRAFRACTION MOTION AND SHALLOW DEPTH DOSE PROFILES ON THE CHOICE OF PTV DEPTH \\ by
}

JAREd H. Strydhorst, B.Sc.

A thesis submitted to the Faculty of Graduate Studies and Research

in partial fulfillment of the requirements for the degree of Master of Science

Department of Physics

Carleton University Ottawa, Ontario

September, 2008

Copyright (C)2008 Jared Strydhorst 


$\begin{array}{ll}\begin{array}{l}\text { Library and } \\ \text { Archives Canada }\end{array} & \begin{array}{l}\text { Bibliothèque et } \\ \text { Archives Canada }\end{array} \\ \begin{array}{l}\text { Published Heritage } \\ \text { Branch }\end{array} & \begin{array}{l}\text { Direction du } \\ \text { Patrimoine de l'édition }\end{array} \\ \begin{array}{l}\text { 395 Wellington Street } \\ \text { Ottawa ON K1A 0N4 } \\ \text { Canada }\end{array} & \begin{array}{l}\text { 395, rue Wellington } \\ \text { Ottawa ON K1A 0N4 } \\ \text { Canada }\end{array}\end{array}$

Your file Votre référence ISBN: 978-0-494-44143-5 Our file Notre référence ISBN: 978-0-494-44143-5

NOTICE:

The author has granted a nonexclusive license allowing Library and Archives Canada to reproduce, publish, archive, preserve, conserve, communicate to the public by telecommunication or on the Internet, loan, distribute and sell theses worldwide, for commercial or noncommercial purposes, in microform, paper, electronic and/or any other formats.

The author retains copyright ownership and moral rights in this thesis. Neither the thesis nor substantial extracts from it may be printed or otherwise reproduced without the author's permission.
AVIS:

L'auteur a accordé une licence non exclusive permettant à la Bibliothèque et Archives Canada de reproduire, publier, archiver, sauvegarder, conserver, transmettre au public par télécommunication ou par l'Internet, prêter, distribuer et vendre des thèses partout dans le monde, à des fins commerciales ou autres, sur support microforme, papier, électronique et/ou autres formats.

L'auteur conserve la propriété du droit d'auteur et des droits moraux qui protège cette thèse. $\mathrm{Ni}$ la thèse ni des extraits substantiels de celle-ci ne doivent être imprimés ou autrement reproduits sans son autorisation.
In compliance with the Canadian Privacy Act some supporting forms may have been removed from this thesis.

While these forms may be included in the document page count, their removal does not represent any loss of content from the thesis.
Conformément à la loi canadienne sur la protection de la vie privée, quelques formulaires secondaires ont été enlevés de cette thèse.

Bien que ces formulaires aient inclus dans la pagination, il n'y aura aucun contenu manquant.

\section{Canada}




\section{Abstract}

When treating breast cancer, radiation therapy is used as an adjuvant to surgery to reduce the likelihood of recurrence. Historically, this has taken the form of static, opposed parallel beams of radiation. Tomotherapy, a relatively new method of delivering radiation therapy, offers the possibility of improved dose conformality and less normal tissue toxicity by using helically-delivered dynamically-modulated beams. In this work, we use four-dimensional computed tomography to confirm the efficacy of a using a thermoplastic shell to reduce the respiratory motion of the breast during tomotherapy. We also investigate the problem of optimizing the dose distribution when the target volume is near the surface of the patient. By creating and delivering treatment to a breast-like phantom we demonstrate the necessity of maintaining a margin of at least $2 \mathrm{~mm}$ between the skin surface and the target volume to achieve acceptable dose homogeneity. 


\section{Acknowledgements}

I would like to express my sincere thanks to my supervisor, Dr. Miller MacPherson, for all of his guidance and input into this research and preparation of this thesis. I would also like to thank Dr. Brenda Clark for her invaluable input into this work and for having extracted from my writing any number of unnecessary phrases, inappropriate adjectives, awkward and wordy constructions and other verbal tics.

The work on immobilization was part of a clinical trial overseen by Dr. Jean-Michel Caudrelier, whose input and feedback were much appreciated.

The contributions of the therapists in the tomotherapy unit at The Ottawa Hospital Cancer Centre are also much appreciated. I would like to thank in particular Lynn Montgomery, Greg Fox and Kathy Carty for their assistance with using the tomotherapy system.

For volunteering to spend many hours in the tedious work of contouring 4DCT slices for the study that makes up a significant portion of this work, I would like to express my thanks to Thomas Souchen.

And for making the graduate students' lab at the cancer centre such an entertaining, albeit not always productive, environment, I extend my undying gratitude to, in no particular order, Amanda Cherpak, Elena Gil, Maria Lourdes Garcia Fernandez, Tara Wood, and Leah Shuparski.

Thank you all. 


\section{Contents}

1 Introduction 1

$1.1 \quad$ Breast Cancer . . . . . . . . . . . . . . . . . 1

1.1 .1 Treatment . . . . . . . . . . . . . . 2

1.1 .2 Tangential Fields $\ldots \ldots \ldots \ldots \ldots \ldots \ldots$

1.2 Treatment Outcomes and Risks . . . . . . . . . . . . 5

1.2 .1 Recurrence . . . . . . . . . . . . . . . . . 5

1.2.2 Radiotherapy induced morbidities $\ldots \ldots \ldots \ldots \ldots$

1.3 Objective of the thesis $\ldots \ldots \ldots \ldots \ldots \ldots \ldots$

2 Tomotherapy 9

2.1 Background $\ldots \ldots \ldots \ldots \ldots \ldots \ldots \ldots \ldots \ldots \ldots \ldots \ldots \ldots \ldots$

2.1.1 Intensity Modulated Radiation Therapy $\ldots \ldots \ldots$. . . . . . 9

2.1.2 Inverse treatment planning $\ldots \ldots \ldots \ldots \ldots \ldots$

2.1.3 Image Guided Radiation Therapy $\ldots \ldots \ldots \ldots$

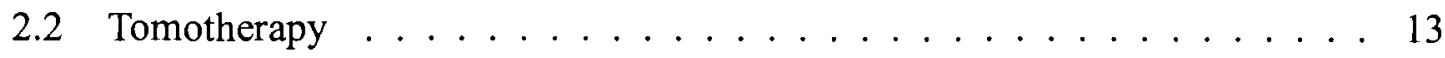


2.2 .1 Treatment Beam . . . . . . . . . . . . . . 13

2.2.2 Treatment Planning . . . . . . . . . . . . . . . 15

2.2.3 Beamlet Calculation . . . . . . . . . . . . . 16

2.2 .4 Optimization $\ldots \ldots \ldots \ldots \ldots \ldots \ldots$

2.3 Tomotherapy and Breast Cancer $\ldots \ldots \ldots \ldots \ldots$

2.3 .1 Advantages . . . . . . . . . . . . . . 22

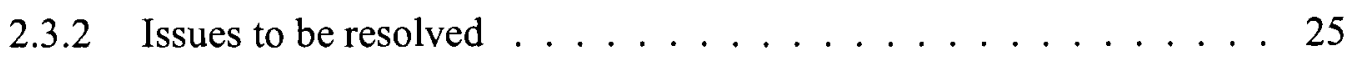

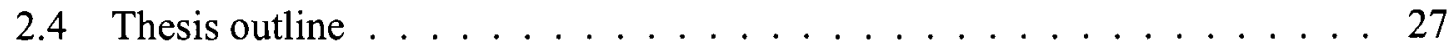

3 Patient Immobilization $\quad 28$

3.1 Breathing Motion $\ldots \ldots \ldots \ldots \ldots \ldots \ldots \ldots \ldots \ldots \ldots$

3.1 .1 Breast Motion $\ldots \ldots \ldots \ldots \ldots \ldots \ldots$

3.1 .2 Lung Motion $\ldots \ldots \ldots \ldots \ldots \ldots$

3.1 .3 Heart Motion . . . . . . . . . . . . . . . . . . . 35

3.2 Materials and Method . . . . . . . . . . . . . 36

3.2 .1 Patients . . . . . . . . . . . . . . . 36

3.2.2 Immobilization and Breath Coaching $\ldots \ldots \ldots \ldots$

3.2.3 4-Dimensional Computed Tomography $\ldots \ldots \ldots \ldots$. . . . 37

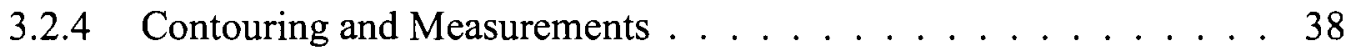

3.2 .5 Shift data analysis $\ldots \ldots \ldots \ldots \ldots \ldots \ldots$

3.3 Results and Analysis $\ldots \ldots \ldots \ldots$. . . . . . . . . . . 43 
$3.3 .1 \quad 4 \mathrm{D} \mathrm{CT} \mathrm{study} \ldots \ldots \ldots \ldots \ldots \ldots$

3.3.2 Pre-treatment Shift Data . . . . . . . . . . . . . 46

3.4 Conclusions . . . . . . . . . . . . . . . . . . 49

4 Dosimetric Consequences of PTV Depth 51

4.1 PTV Depth and Buildup Region . . . . . . . . . . . . . . . . 51

4.2 Materials and Method . . . . . . . . . . . . . . . 56

$4.2 .1 \quad$ EDR2 Film . . . . . . . . . . . . . 56

4.2 .2 Gafchromic Film . . . . . . . . . . . . . . . 60

4.2 .3 Treatment Planning . . . . . . . . . . . . . . . 60 60

4.2.4 CT data to relative mass density conversion . . . . . . . . . . 61

4.2 .5 Measurements . . . . . . . . . . . . . . 68

4.3 Results and Analysis . . . . . . . . . . . . . . . . 72

4.3.1 Density Conversion Table . . . . . . . . . . . . . 72

4.3.2 Effect of PTV depth on dose profile . . . . . . . . . . 75

4.3.3 Surface Dose Measurements . . . . . . . . . . . . . . 81

4.3.4 Sensitivity of dose profile to misalignment of phantom . . . . . . 83

4.4 Conclusions . . . . . . . . . . . . . . . . . . . . 86

4.4.1 CT number to mass density curve . . . . . . . . . . 86

4.4.2 PTV Depth and Dose Dumping . . . . . . . . . . . 86

4.4.3 Sensitivity to Misalignment . . . . . . . . . . . . 87 
5 Conclusions $\quad 89$

5.1 Immobilization . . . . . . . . . . . . . . . 89

5.2 PTV Depth $\ldots \ldots \ldots \ldots$. . . . . . . . . . . . . . 91

5.3 Recommendations . . . . . . . . . . . . . . . 91 


\section{List of Tables}

3.1 Maximum displacement during free-breathing at the mid-breast level for a patient immobilized with a thermoplastic shell . . . . . . . . . . . . 44

3.2 Displacement $51 \mathrm{~mm}$ superior to mid-breast level . . . . . . . . . 45

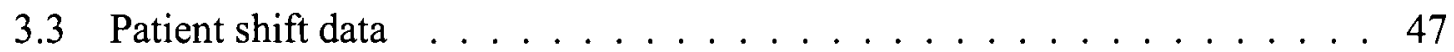

4.1 Optimization parameters entered into the tomotherapy planning software for the planning target volume (PTV) and organ at risk(OAR) . . . . . 63

4.2 Surface dose predictions and Gafchromic measurements . . . . . . . 83 


\section{List of Figures}

1.1 Conventional breast irradiation with tangential fields $\ldots \ldots \ldots$

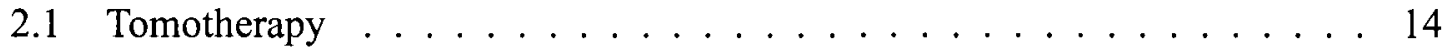

2.2 Superposition in regions of lateral electronic disequilibrium. . . . . . . . . 20

3.1 Lung and skin contours showing maximum displacement over breathing cycle. ........................... 41

4.1 Calibration film. ................... 58

4.2 Optical density profile of calibration film along the $\mathrm{X}$ axis. Pixel values are in arbitrary scanner units. . . . . . . . . . . . . . . 58

4.3 Treatment planning contours ................... 62

4.4 Plot of the CT number-to-relative mass density conversion table. . . . . . 65

4.5 Histogram of the CT values for 500000 voxels containing air. . . . . . . 67

4.6 Tomophantom with film . . . . . . . . . . . . . . . . . . . . . 69 
4.7 Calculated and measured dose profiles for unmodified and modified relative density tables. . . . . . . . . . . . . . . 73

4.8 Calculated and measured dose distributions in the X-Y plane . . . . . . 76

4.9 Dose profiles in the $\mathrm{X}$ and $\mathrm{Y}$ directions for a PTV depth of $0 \mathrm{~mm} \ldots \ldots$

4.10 Dose profile along $X$ axis at centre of phantom for PTV depth of $2 \mathrm{~mm}$. . . 79

4.11 Dose profile along $X$ axis at centre of phantom for PTV depth of $4 \mathrm{~mm}$. . . 79

4.12 Dose profile along $X$ axis at centre of phantom for PTV depth of $6 \mathrm{~mm}$. . . 80

4.13 Dose profiles along $X$ axis at centre of phantom for PTV depth of $10 \mathrm{~mm}$. . 80

4.14 Dose profile parallel to axis of gantry at depths of $5 \mathrm{~mm}$ and $50 \mathrm{~mm}$. . . 82

4.15 Dose profiles showing the consequences of misaligning the phantom . . . 85 


\section{Glossary}

adjuvant used in addition (to another type of treatment)

BCS breast conserving surgery

bolus tissue equivalent material, usually wax, placed on the surface of the skin to allow delivery of full dose at the skin surface

contralateral on the opposite side of the body, relative to the breast being treated

CTV clinical target volume; an volume containing the GTV plus any tissue likely to contain subclinical disease

DVH dose-volume histogram; a histogram displaying the percentage of an organ or other defined structure receiving a particular dose

flash in conventional breast radiation therapy, an expansion of the treatment beams into the air in front of the breast to allow for motion and setup error

GTV gross tumour volume; the volume of tissue containing visible disease 
IGRT image guided radiation therapy

IMRT intensity modulated radiation therapy

ipsilateral on the same side of the body as the breast being treated

lumpectomy surgical removal of cancerous tissue from a breast

mastectomy surgical removal of all breast tissue from a breast

MLC multi-lead collimator

parenchyma the functional tissue of an organ, in the breast it refers to the glandular tissue

PTV planning target volume; defined as the CTV plus the margins necessary to guarantee full dose is delivered to the entire CTV

TERMA total energy released pre unit mass; total energy released by photon interactions in a finite volume of matter divided by the mass of that volume 


\section{Chapter 1}

\section{Introduction}

Each year, over 150,000 Canadians are diagnosed with cancer ${ }^{1}$. Over 70,000 will die from the disease. As the population grows and ages, this number will continue to rise, even with no change in the age-adjusted incidence rate.

\subsection{Breast Cancer}

Breast cancer is the most common form of cancer diagnosed in women, affecting about 1 in 9 women at some point in their life ${ }^{1}$.

By definition breast cancer is any cancer that originates in the breast tissue; left untreated it can metastasize and spread to other organs in the body via the blood or the lymphatic system. Breast cancer is fatal in approximately one third of women diagnosed with the disease. 


\subsubsection{Treatment}

\section{Surgery}

Almost all women diagnosed with breast cancer will undergo surgery to remove the tumour. Until the 1970s, breast cancer was nearly always treated by mastectomy-removal of the entire affected breast and surrounding muscle and fascia. Today, provided the cancer is diagnosed relatively early, treatment more frequently involves lumpectomy-breast conserving surgery (BCS) comprising removal of the visible tumour that spares the healthy breast tissue. When the cancer is more advanced, a total mastectomy may still be indicated. Cosmetic outcome makes BCS preferable to a full mastectomy, but without adjuvant treatment the risk of recurrence is higher with BCS.

Surgical excision of the cancerous tissue is often followed by adjuvant therapy, either chemotherapy or radiation therapy or a combination of the two, aimed at killing any cancer cells left behind following surgery. When used in combination with adjuvant therapy, the risk of recurrence following BCS is comparable to that for a full mastectomy ${ }^{2}$.

\section{Radiation therapy}

Radiation therapy is the use of radiation to destroy living tissue. It uses ionizing radiation to transfer enough energy to electrons within living cells to damage the biomechanical machinery comprising the cells. In particular, the radiation damages the cells' internal 'blueprints' — the DNA — preventing the cell from repairing itself or reproducing. Cancer 
cells are normal cells that have mutated in some way that prevents them from carrying out their normal functions, and in particular, from stopping cell division once they have reached a certain limit. In some cases, the mutations responsible for the cancer also impair the selfrepair mechanisms of the cell. If a cancerous cell and a healthy cell are both damaged to the same extent, the cancerous cell will often (though not always) be less successful in repairing itself than a healthy cell.

Statistically, the relationship between the radiation dose delivered to living tissue and the fraction of the cells killed will (hypothetically) follow a sigmoidal curve. At low doses, cells are able to repair the vast majority of the damage and carry on essentially undisturbed. Beyond some threshold, more and more cells will be unable to repair the damage. Beyond a certain limit, nearly all the cells will be killed. The success of radiotherapy depends, in part, on the cancerous cells being more radiation sensitive than healthy tissue. If this is the case, a uniform dose of radiation to a volume of tissue containing both healthy and cancerous cells would be able to kill the cancer, while only partially damaging the healthy tissue. However, it is unlikely that the radiation sensitivity would differ radically enough that treatment could be as straightforward as delivering a single dose that would be lethal to cancer but not to normal tissue. In general the difference in radiation sensitivity is small, and even then there would be no way to calculate exactly what dose would achieve the optimum balance between killing cancer and sparing healthy tissue. This problem can be dealt with by fractionating the radiation treatment.

Fractionation involves daily treatment of the affected tissue with a dose large enough 
to cause damage, but low enough that most healthy cells should be capable of repairing themselves in a short period of time. Cancer cells will also undergo self-repair, though hopefully to a lesser degree because their mutations have impaired their repair mechanism. By repeatedly treating the patient with the same dose over a period of 20-30 days, the hope is that the healthy tissue will be able to keep up with repairing the radiation damage, while the damage to the cancerous tissue accumulates until the cancerous cells have been destroyed.

For breast cancer, radiotherapy is used in addition to other methods of cancer treatment, such as chemotherapy and surgery. Because the visible tumour has already been surgically excised, the goal is usually to treat the remaining breast tissue to kill any cancerous cells not part of the tumour mass or not removed by surgery. Surgical removal of a tumour also has the potential for 'seeding,' the deposition of cancerous cells elsewhere within the surgical scar. Therefore, special attention is paid to including the surgical scar in the target during adjuvant radiotherapy.

For women diagnosed with ductal carcinoma in situ (DCIS), generally considered a precursor for invasive carcinoma, the rate of disease-free survival is significantly better for women who undergo adjuvant radiotherapy in addition to breast conserving surgery (BCS) compared to those who undergo surgery alone ${ }^{3}$. Likewise, other studies have reported a survival advantage for women with more advanced disease (stage II or III) who receive adjuvant radiation therapy in addition to mastectomy ${ }^{4,5}$ or in addition to $\mathrm{BCS}^{6}$.

The presence of cancerous cells within the lymph nodes is indicative of more advanced 
disease and correlated with greater odds of recurrence and a lower probability of survival. Where nodal involvement is demonstrated or suspected, the lymph nodes connected with the breast will also be irradiated.

\subsubsection{Tangential Fields}

Historically, most radiation therapy for breast cancer has been treated using tangential fields, shown in Figure 1.1. If the involvement of the locoregional nodes is suspected, this entails a four-field technique that uses two fields to cover the breast and axillary nodes and anterior and posterior parallel opposed fields to treat the clavicular nodes. Wedge filters are used to improve dose uniformity, but they have the disadvantage of only permitting a dose gradient in a single direction. Better dose uniformity can be achieved with intensity modulated radiation therapy (IMRT, described in more detail in chapter 2), which allows for more complex shaping of field intensity than a wedge.

\subsection{Treatment Outcomes and Risks}

\subsubsection{Recurrence}

Recurrence is the most significant long-term risk faced by a patient who has undergone treatment for breast cancer. Underdosing cancerous tissue or missing some cancerous cells entirely increases the likelihood that the cancer will eventually return either locally or as 


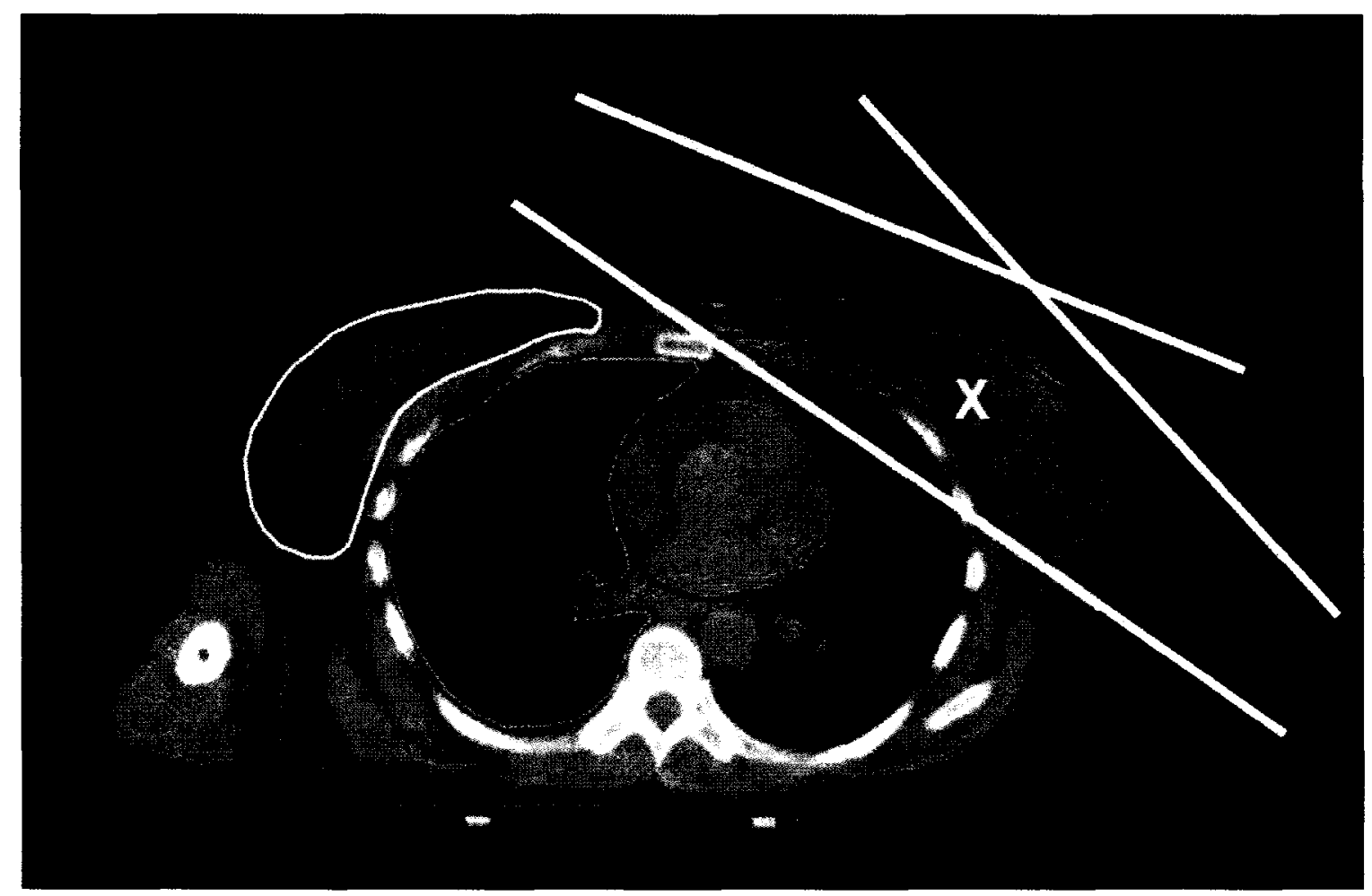

Figure 1.1: Conventional breast irradiation delivers dose to the breast tissue, outlined in red, using two tangential radiation fields, delineated by the heavy white lines. ' $\mathrm{X}$ ' marks the isocenter. 
distant metastases.

\subsubsection{Radiotherapy induced morbidities}

All exposure to ionizing radiation carries with it some risk of inducing or contributing to the development of cancer ${ }^{7}$. Furthermore, acute doses of radiation can also induce other non-cancerous complications. Dose to the lungs, for instance, can cause pneumonitis and pulmonary fibrosis. Most significantly for breast cancer treatment, excess radiation can damage the heart, potentially contributing to the development of atherosclerosis and late cardiac morbidity. One study has reported a cardiovascular disease hazard ratio of 1.27 ( $95 \%$ confidence interval $1.07-1.51 ; \mathrm{p}=0.006$ ) for irradiated vs. non-irradiated women treated for right-sided breast cancer ${ }^{8}$. The same study also showed increased mortality from cardiovascular disease for women treated for left-sided vs. right-sided breast cancer (hazard ratio $1.25 ; 95 \%$ confidence interval $1.05-1.49 ; \mathrm{p}=0.014$ ).

Many women who undergo radiation treatment for breast cancer also suffer from painful skin conditions caused by the radiation. Pain, erythema, edema, hyperpigmentation, and skin desquamation are all potential side effects. When treating tumours deep within the body, such as a prostate tumour, the treatment beams enter the body more or less perpendicular to the skin. Because of the distance required to establish charged particle equilibrium the dose to the skin remains relatively low, an effect known as skin sparing. Breast radiation therapy as described above uses beams tangential to the patient and beams that in some places, particularly in the nipple area and near the lateral and medial borders of the 
breast, exit the skin near the point of maximum dose, increasing the dose to the skin and the associated side effects.

\subsection{Objective of the thesis}

Tomotherapy is a relatively new tool designed to deliver highly conformal, image-guided radiation therapy using a vertical ring gantry containing a rotating megavolt accelerator. This equipment has been shown to substantially improve dose conformality to complex shaped targets thereby reducing normal tissue dose but, to date, the application of tomotherapy to breast cancer has been limited and there remain several issues to be resolved. The rationale for the use of tomotherapy for the treatment of breast cancer is to reduce the toxicity of the current treatments, in particular the occurrence of damage to the skin. The objectives of this work are to investigate the management of respiratory motion and the management and accuracy of dose in proximity to the skin for irradiation of the breast and associated lymph nodes. This work is expected to resolve these outstanding issues to enable the use of tomotherapy for the treatment of breast cancer. 


\section{Chapter 2}

\section{Tomotherapy}

Tomotherapy, a radiation therapy technique developed at the University of Wisconsin, is a relatively new method of delivering highly conformal radiotherapy for treating cancer. The system combines intensity modulated radiation therapy (IMRT) with image-guided radiation therapy (IGRT) ${ }^{9}$.

\subsection{Background}

\subsubsection{Intensity Modulated Radiation Therapy}

In conventional radiotherapy, dose is delivered to the tumour using fields of uniform intensity shaped to conform to the tumour and avoid sensitive organs. This delivers dose to not only the tumour, but also to the rest of the tissue within the radiation field, both in front of and behind the tumour. By treating using multiple beams, each delivered from a different 
angle, the geometry of the beams can be set up so that the target receives dose from every beam, while the surrounding tissue only receives dose from the one or two beams that pass through to treat the tumour.

However, this method has some significant shortcomings. Foremost among these is the inability to conform dose to a concave target. This difficulty increases as the complexity of the shape of the target increases. The situation becomes more complicated with variations in the volume and density of tissue between the radiation source and the tumour.

Moreover, some structures in the body, for example the heart or spinal cord, are more sensitive to radiation and are best avoided altogether. When a beam is delivered from an angle where such a structure is in the way, the radiation field may be blocked to avoid damage to the organ at risk. As a consequence, this field will only partially cover the target volume.

IMRT improves both the uniformity of the coverage and the ability to deliver complex dose distributions while avoiding sensitive structures. It does this by using fields of nonuniform intensity. IMRT compensates for the fact that some beams provide only partial coverage of the target by increasing the intensity of portions of other fields that treat those areas that the first field doesn't.

Calculating the particular combination of field intensities needed to deliver the optimal dose is non-trivial. The treatment planner is faced with a challenge involving a bewildering number of degrees of freedom, since, within certain restrictions imposed by the ability of the treatment system to deliver dose, each radiation field is permitted to assume an arbitrary 
distribution of intensities. The total dose delivered is a summation of the doses delivered by all of the individual fields, and changing the intensity pattern delivered by one field will alter the doses that must be delivered by all the other fields.

\subsubsection{Inverse treatment planning}

The solution to this challenge is to pose it as an optimization problem, where the goal is to solve for the particular set of fields required to give the requested dose distribution.

Historically, this process has been given the name "inverse treatment planning", in contrast to forward treatment planning where the planner would specify the beams to be used, then verify that the target was adequately covered.

Instead of specifying beams, the planner specifies target volumes and avoidance structures. For each of these, penalty factors are chosen for overdosing, and, in the case of the target volume, under-dosing the tissue in question. Planning software may also allow the planner to specify either a minimum point on the dose-volume histogram (DVH) curve for a target volume, or a maximum point on the DVH curve for an avoidance structure.

Usually, the treatment planner will also select the gantry angles based on a standard treatment geometry for the location of the tumour. Selection of the intensity patterns is then up to the treatment planning system software. Optimization software evaluates thousands of potential fluence patterns, each time comparing the resulting dose distribution to that requested by the planner, and deciding, based on the penalty weighting assigned, whether the plan is an improvement on the last one. Based the results, the plan is modified and 
recalculated until the resulting dose distribution is deemed to be acceptable. In practice, the approach taken by the software may diverge substantially from this description, but the essential point remains: inverse planning uses software to define the fluence of each beam to create a plan that will deliver a dose distribution to the target volume.

\subsubsection{Image Guided Radiation Therapy}

The ability to deliver highly conformal doses means that higher doses may be prescribed to a tumour, while sparing a sensitive structure nearby. However, delivering high doses in the vicinity of sensitive structures also makes the clinical outcome more sensitive to accurate alignment. This is of particular concern when a treatment is delivered over the course of 20-30 days spread out over a period of several weeks. The difficulty of placing the patient in exactly the same position each day is compounded by the likelihood that a patient will gain or (more likely) lose weight or that the tumour may shrink over the course of the treatment.

IGRT improves the repeatability of the patient alignment by imaging the patient before delivering each treatment fraction. This allows the radiation therapist delivering the treatment to make any necessary adjustments to align the target within the beam. Various methods-for example ultrasound, portal imaging, or cone-beam CT—can can used. Incorporating the imaging equipment into the treatment system itself means that the patient will be moved as little as possible between the alignment scan and treatment. 


\subsection{Tomotherapy}

The geometry of the tomotherapy system is identical to a CT scanner, consisting of an x-ray source mounted in a ring gantry that rotates around the patient. As the source rotates, the couch moves the patient through the bore of the unit. Thus, the radiation source follows a helical path around the patient, exactly as in a CT scanner. Treating a patient requires x-rays of much higher energies than those needed to scan a patient. Instead of an x-ray tube, tomotherapy uses a small linear accelerator to generate the treatment beam (Figure 2.1).

\subsubsection{Treatment Beam}

Tomotherapy uses a fan shaped beam to deliver dose to the patient. The linear accelerator used to produce the treatment beam is capable of accelerating electrons to an energy of $6 \mathrm{MeV}$.

Conventional radiation therapy systems use a flattening filter to produce a treatment beam with a uniform intensity profile. However, this also reduces the overall intensity of the beam, requiring longer treatment times. To minimize treatment times, tomotherapy, unlike most radiation therapy treatment systems, does not use a flattening filter. This results in a beam with cone shaped intensity profile. Along the y-axis (parallel to the bore of the gantry), the width of the beam can be set by the y-jaw collimator to $1,2.5$, or $5 \mathrm{~cm}$. Along the width of the fan, perpendicular to the axis of the bore, the intensity can be 


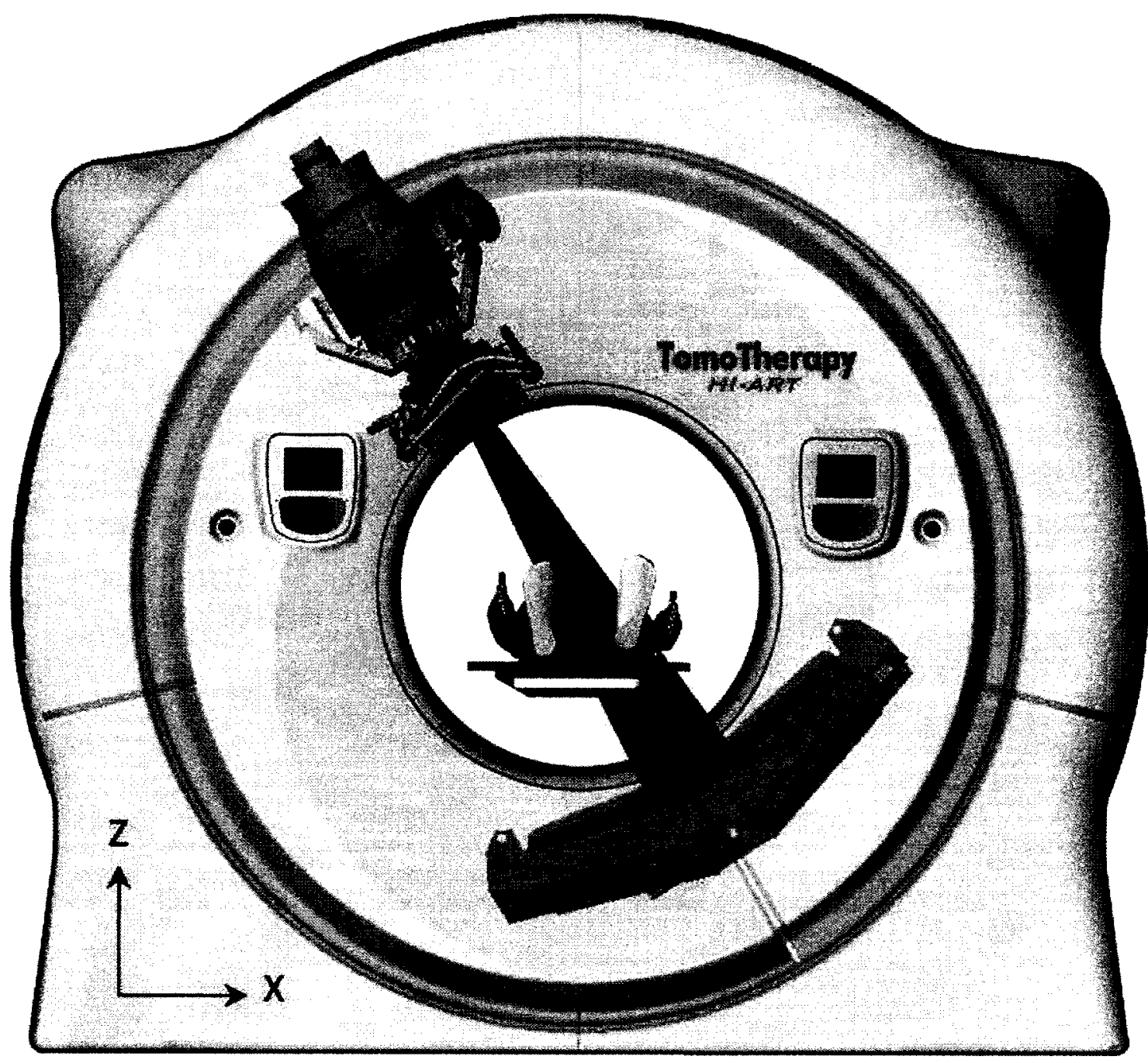

Figure 2.1: Tomotherapy system showing the position of the linear accelerator, multi-leaf collimator, treatment beam, and imaging detector. Figure courtesy of TomoTherapy Inc. 
continuously modulated by a binary multi-leaf collimator. The resulting intensity profile will be the product of the underlying cone-shaped profile of the beam and the open-time of the respective multi-leaf collimator leaves.

An array of sensors opposite the beam source allows the same linear accelerator used for treatment to also function as part of a megavoltage CT scanner to facilitate image guided radiation therapy. To scan the patient, the linear accelerator is detuned to reduce the beam energy to approximately $3 \mathrm{MV}$.

The multi-leaf collimator (MLC) used in tomotherapy comprises sixty-four tungsten leaves which divide the fan beam into sixty-four segments along its width, each with projected width of $6.25 \mathrm{~mm}$ at isocenter. Each leaf is controlled independently by a pneumatic actuator. Control of the leaves is binary; at any given time a single leaf can be either open or closed. By modulating the length of time that each MLC leaf is open for each projection, the intensity of each segment of the fan beam can be modulated.

\subsubsection{Treatment Planning}

The process of creating a tomotherapy treatment plan for a patient starts with obtaining a 3D CT scan of the patient containing the volume to be treated. The oncologist then contours the CT slices of the patient, delineating the gross tumour volume (GTV). This is then expanded to include tissue suspected of containing subclinical disease resulting in a clinical target volume (CTV). The oncologist also specifies any critical structures to be avoided. 
The treatment planner then expands the CTV to account for patient movement and misalignment and other uncertainties in the targeting of the beam to create the planning target volume (PTV).

For each PTV, a prescription dose is assigned as prescribed by the oncologist. Dose limits are assigned to each avoidance structure. For both the PTV and the organs at risk, various dose penalties are also specified, as described below in section 2.2.4.

\subsubsection{Beamlet Calculation}

To make the problem of calculating the dose to the patient manageable, the treatment beam is divided into 'beamlets'. Each full gantry rotation is divided into 51 discrete projections, spaced about $7^{\circ}$ apart. The fan beam is divided into 64 segments corresponding to the 64 leaves of the multi-leaf collimator. Therefore, for each gantry rotation there are over 3000 possible beamlets. The number of rotations required for a given treatment plan will vary depending on the extent of the target to be treated, the width chosen for the treatment beam and the rate at which the patient is moved through the bore during treatment. Typically, a plan may contain between $10-30$ rotations - potentially more than 100,000 possible beamlets.

Beamlet calculation is the process of the calculating the dose delivered to each voxel of the patient by each MLC projection. To perform the necessary calculations, the data from the CT scan of the patient is converted to a three dimensional matrix of voxels, each with a particular mass density. To reduce the total amount of calculation required, those beamlets 
which don't actually pass through any of the target volumes are eliminated from the list of possible beamlets to be used by the treatment plan.

Tomotherapy beamlet calculation uses a superposition algorithm to calculate the dose that would be delivered to each voxel by each beamlet. This is a two step approach which first calculates the total energy transferred to the medium by photon interactions in each voxel. This is expressed as total energy released per unit mass (TERMA). A dose spread function describes how the TERMA from a particular voxel gets deposited as dose in the surrounding voxels. The final dose distribution is calculated by convolving the TERMA for each voxel with this dose spread function.

The distribution of the TERMA within the volume of the patient can be calculated relatively efficiently. TERMA corresponds to photon interactions and photons travel in straight lines and are attenuated only by the material through with they pass.

The path of an energetic electron and the way it will deposit energy is more complex. In practice, tomotherapy uses energy deposition kernels that have been pre-calculated using Monte Carlo simulation to determine how the energy from a unit amount of TERMA in a particular voxel is distributed to all of the surrounding voxels. These energy deposition kernels are calculated for water. To use them to calculate dose in an actual patient, various factors must be taken into account, including the angle of the incident photons (dealt with by kernel tilting) and the energy spectrum of the photons incident on a particular volume (which requires the energy deposition kernels to be calculated for all possible depths). The kernels are also scaled according to the mass density of the tissue through which they pass 
to account for inhomogeneities. Mathematically, this convolution of TERMA with a scaled energy deposition kernel can be expressed as follows ${ }^{10}$ :

$$
D(\mathbf{x})=\frac{\iiint T(\mathbf{s}) \rho(\mathbf{s})\left[\eta(\mathbf{x}) \bar{\eta}^{2} h(\mathbf{r}(\mathbf{s}, \mathbf{x})) \Delta V\right] d V_{s}}{\rho(\mathbf{x}) \Delta V}
$$

where

$D(\mathbf{x})$ dose to point $\mathbf{x}$

s position of source (TERMA) point

$T$ TERMA

$\rho$ density

$\eta$ density relative to water

$\bar{\eta}$ mean relative density between $\mathbf{s}$ and $\mathbf{x}$

$h$ normalized energy deposition kernel

$\mathbf{r}(\mathbf{s}, \mathbf{x})$ radiological distance from $\mathbf{s}$ to $\mathbf{x}$

$\Delta V$ destination volume

The kernel is scaled using the radiological distance, rather than the geometrical distance, as the argument in $h$, the function describing the normalized energy deposition kernel. The convolution algorithm breaks down in regions of lateral electronic disequilibrium. Considering the situation depicted in Figure 2.2, it can be seen that the calculated dose to point $\mathbf{x}$ per unit of TERMA at point $\mathbf{s}$ will be the same as the dose to point $\mathbf{x}^{\prime}$ per unit of TERMA at point $\mathbf{s}^{\prime}$. This must be the case, since the distance and direction from $\mathbf{s}$ to $\mathbf{x}$ is the same as from $\mathbf{s}^{\prime}$ to $\mathbf{x}^{\prime}$, and since the region has uniform density, the radiological distances 
( $\mathbf{r}$ and $\mathbf{r}^{\prime}$ ) are the same in both cases. However, the dose is actually deposited by energetic electrons which follow convoluted paths from the TERMA point to the dose deposition point. Therefore, some of the dose to point $\mathbf{x}$ has presumably been deposited by electron scattered in from the right. In the case of point $\mathbf{x}^{\prime}$ however, any electrons that scatter into the low density region are extremely unlikely to be scattered back into the high density, and $\mathbf{x}^{\prime}$ will in fact absorb less dose than predicted. More succinctly, the dose scaling used in the convolution algorithm looks only at the path between the TERMA point and the dose deposition point and doesn't consider the adjacent material which will have an effect on dose distribution. This effect has been reported by Jones and Das ${ }^{11}$.

\subsubsection{Optimization}

The dose delivered to voxel $i, D_{i}^{d}$ then is given by

$$
D_{i}^{d}=\sum_{j} d_{i j} w_{j}
$$

where $d_{i j}$ is the dose to the $i$ th voxel by beam $j$, and $w_{j}$ is the weighting (intensity) of beam $j$.

Optimization is the process of selecting the set of beam weightings necessary to achieve the desired dose distribution. 


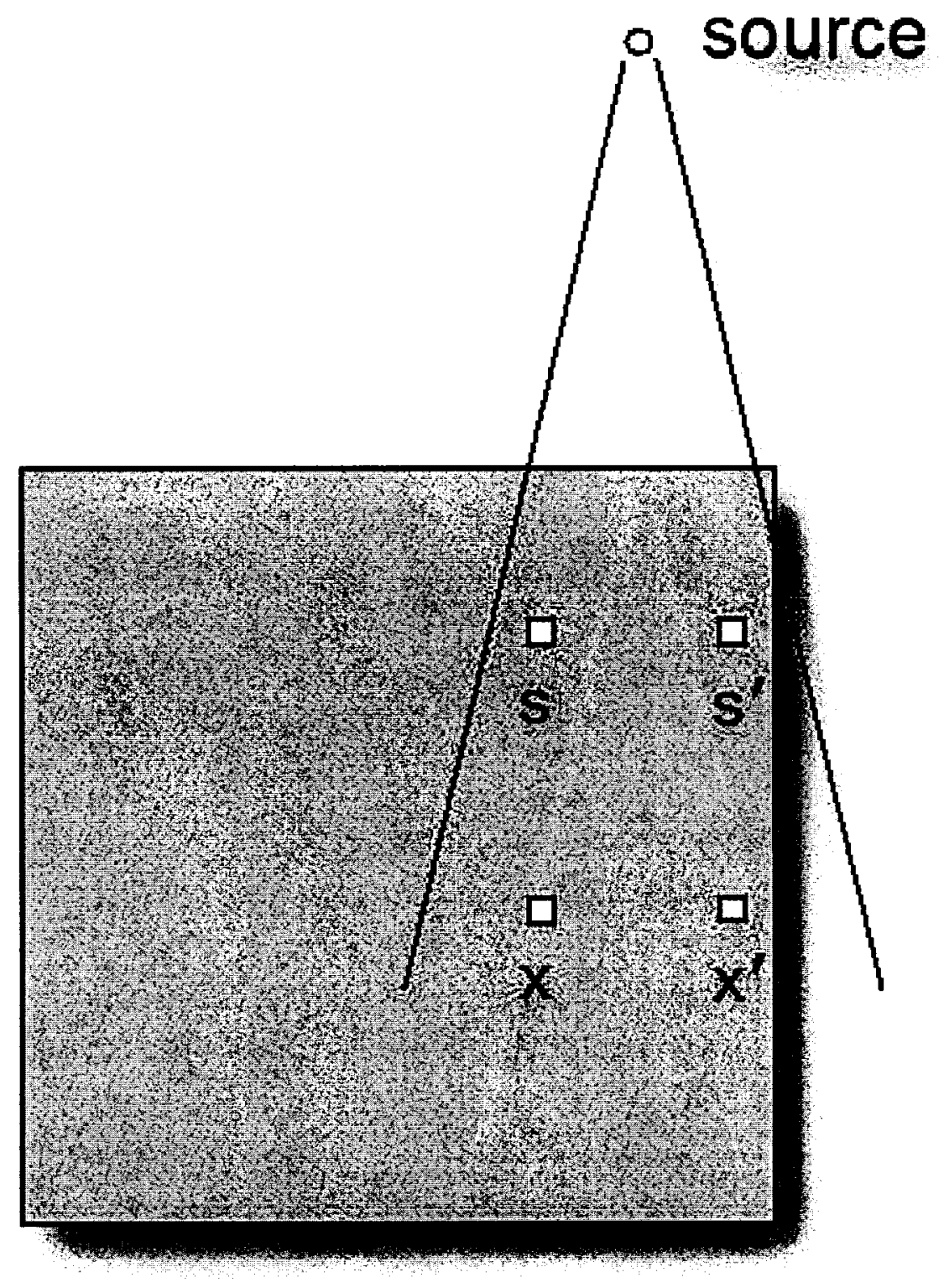

Figure 2.2: Superposition in a region of lateral electronic disequilibrium. The shaded region corresponds to tissue (or any other dense medium). Surrounding region contains air. Points $\mathbf{s}$ and $\mathbf{s}^{\prime}$ indicate TERMA source points, $\mathbf{x}$ and $\mathbf{x}^{\prime}$ are the points at which the dose is calculated. 
Tomotherapy uses a least-squares optimization method with the goal of minimizing the following objective function:

$$
O(\vec{\Psi})=\sum_{T} \sum_{i \in t} \frac{\alpha_{t} \beta_{t_{i}, \max } \beta_{t_{i}, \min }}{N_{t}}\left(D_{i}^{p}-D_{i}^{d}\right)^{2}+\sum_{R} \sum_{i \in r} \frac{\alpha_{r} \beta_{r_{i}, \max } \beta_{r_{i}, D V H}}{N_{r}}\left(D_{i}^{p}-D_{i}^{d}\right)^{2}
$$

where

$\vec{\Psi} \quad$ is the vector of all beam weightings, $w_{1}, w_{2}, \ldots$

$T$ summation over all targets

$R \quad$ summation over all avoidance regions

$i \in t \quad$ summation over all voxels in target $t$

$i \in r$ summation over all voxels in avoidance region $r$

$\alpha \quad$ importance of target or avoidance region

$\beta$ dose penalty

$N$ number of voxels in target or avoidance region

$D_{i}^{p} \quad$ dose prescribed to voxel $i$

$D_{i}^{d} \quad$ dose delivered to voxel $i$

The user can guide the optimization by modifying the various parameters in the equation. Changing the values of the $\alpha$ 's allows the user to modify the relative importance of the various targets and avoidance structures. For voxels within a target volume, the $\beta$ parameters in equation 2.3 impose additional penalties on the objective function if the dose delivered is too high or too low. The value of the $\beta$ parameter depends the predicted dose. For example, if the predicted dose to voxel $i$ is less than the maximum dose assigned to 
target $t, \beta_{t, \text { max }}$ is unity, but once the dose to voxel $i$ exceeds the specified maximum dose, $\beta_{t_{i}, \max }$ takes on the value of the maximum dose penalty specified by the user. Likewise, $\beta_{t_{i}, \text { min }}$ takes on the value of the minimum dose penalty if the dose to voxel $i$ falls below the minimum dose assigned to target $t$. Similarly, the $\beta$ parameters impose additional penalties if the dose to an avoidance region is too high or if the DVH curve for that region goes above a specified point.

\subsection{Tomotherapy and Breast Cancer}

\subsubsection{Advantages}

\section{Improved dose conformality and homogeneity}

One advantage of tomotherapy is its ability to deliver highly conformal doses to concave treatment volumes. For breast cancer this may be an advantage, especially where the curvature of the chest wall makes it impossible to use tangential fields without delivering nearly full dose to part of the ipsilateral lung. The downside is a small dose to a larger volume of the lung and other organs at risk. When it comes to dose to organs at risk, the question of 'a little to a lot or a lot to a little' is still controversial.

Research and clinical experience with conventional treatments have demonstrated the presence of 'hot spots' at the lateral and medial borders of the breast and in the nipple area. This dose inhomogeneity is strongly correlated with breast volume and is greater in 
large-breasted women ${ }^{12}$. Wedges and intensity modulation have been used successfully to reduce hot spots, and tomotherapy should also be capable of delivering dose to the breast with even better uniformity.

\section{Minimization of setup error}

The CT scanner integrated into the treatment system allows the patient to be scanned prior to each treatment. This allows misalignments of the patient to be rectified prior to delivering treatment. Typically, a patient may need to be moved by several mm along any axis. A more detailed and quantitative look at the realignment necessary when treating breast cancer follows in Chapter 3 which deals with patient motion, but, in principle, daily CT-based patient alignment may be used to reduce dose to organs at risk by allowing smaller PTVCTV margins to be used in planning.

\section{Irradiation of internal mammary chain}

The ability to deliver highly conformal dose to a precise location using tomotherapy may allow for more effective treatment of the hypothetical subset of patients who would benefit from irradiation of the internal mammary lymph nodes (IMC).

Irradiation of the internal mammary lymph nodes is controversial. Patients who are axillary node-positive and have medially or centrally located tumours have been reported to be more likely to also have IMC involvement. In this case, there may be a survival advantage if the IMC is included in the target to be irradiated. The inner mammary chain 
is located below the ribs, next to the sternum; the IMC associated with the left breast lies directly over the heart.

Most research into the benefits of including the IMC in the PTV has suggested that irradiation of the IMC has little impact on either the development of distant metastases or 15-year overall survival ${ }^{13,14}$. However, prior to routine $\mathrm{CT}$ imaging before treatment, and therefore for many of the patients included in the studies referenced, IMC irradiation was defined as the inclusion within the isodose curve of a point $3 \mathrm{~cm}$ below the skin surface and $3 \mathrm{~cm}$ from the midline towards the ipsilateral side. As the authors of one of those studies note, this approach may potentially miss or underdose the IMC in approximately $17 \%-19 \%$ of patients. Furthermore, using deep tangents may also increase the heart dose, masking any survival benefit of irradiating the IMC by increasing cardiac mortality.

Because tomotherapy allows for more conformal dose distributions than are possible using wide angle tangent fields, it may be possible to irradiate the IMC on some women with less heart dose than could be achieved using conventional treatment methods. (However, because of the nature of tomotherapy, a larger volume of the heart will receive a low dose of radiation.) At least one planning study that compared IMRT with non-IMRT for treatment of the left breast and IMC showed better target coverage and lower normal tissue complication probabilities with IMRT than with wide angle tangents ${ }^{15}$. 


\section{Reduced skin toxicity and improved cosmesis}

Like conventional breast radiation therapy, tomotherapy delivers dose using beams that enter the skin at oblique angles. Measurements made with static tomotherapy beams have shown that skin dose increases significantly as the angle of incidence increases relative to normal incidence ${ }^{16}$.

However, it has also been reported that (non-tomotherapy) intensity-modulated radiation therapy has resulted in significant decreases in skin toxicity compared to conventional techniques ${ }^{17}$.

\subsubsection{Issues to be resolved}

\section{PTV-CTV margins}

Clinical target volume (CTV) is defined as the gross tumour volume plus any tissue suspected to contain sub-clinical disease. In the case of breast cancer, the tumour itself has already been surgically excised and the CTV is conventionally defined as the entirety of the breast parenchyma. To account for setup error and patient motion during treatment, the CTV must then be expanded to guarantee that all of the CTV receives the prescribed dose. This planning target volume is then defined as the target during treatment planning. The amount by which the CTV must be expanded to create the PTV depends on several factors, most importantly the combined uncertainty in patient position as a result of setup error and intrafraction motion. 
Since the patient will be breathing during treatment and may not be positioned exactly the same way each time, ICRU 50 guidelines recommend extending the treatment beam into the air $2 \mathrm{~cm}$ in front of the breast. This expansion of the treatment beam is referred to as 'flash'. With forward treatment planning it is easy to extend the treatment beams beyond the PTV, but with inverse treatment planning, and particularly with tomotherapy where the gantry is continually in motion, extending the beams into the air beyond the patient is problematic. The low density of the air makes it impossible to achieve electronic equilibrium within the air volume, and it is therefore impossible to deliver full dose to the air. Therefore, attempting to add flash within the planning system by expanding the target into the air creates a physically unrealizable target dose distribution that will result in a suboptimal treatment plan with hot spots near the surface of the patient ${ }^{18}$.

Tomotherapy is already known to achieve acceptable dose distributions for targets well below the surface of the skin. The work will investigate the dosemetric consequences of extending the target volume close to the skin surface.

The intent is ultimately to determine where best to define the PTV with respect to the surface of the patient to achieve optimal treatment outcomes.

\section{Dose calculation accuracy in regions of lateral electronic disequilibrium}

The theoretical justification for expecting that the treatment planning system will overestimate the dose delivered in regions of high lateral electronic disequilibrium has already been discussed in section 2.2.3. The clinical significance of this effect remains an open 
question. Part of the aim of this research is to compare the calculated dose to the measured dose for superficial depths where this phenomenon may impact treatment outcome.

\subsection{Thesis outline}

In treatment planning for IMRT, the treatment planner defines the planning target volume (PTV) on a static CT image of the patient, and software then generates a treatment plan that will deliver the prescribed dose to the PTV. For optimal treatment outcome, this planning target volume must be defined so that the resulting plan will deliver the prescribed dose to the entire clinical target volume in spite of the anticipated motion and misalignment of the patient during treatment. In Chapter 3 of this work we measure the geometric uncertainties in patient position from intrafraction motion when the patient is immobilized as per the tomotherapy breast protocol at The Ottawa Hospital Cancer Centre. In Chapter 4 we investigate the dosimetric consequences of the placement of the PTV itself. Together, the outcome of these two investigations will contribute to the establishment of guidelines for target definition that will result in a plan capable of adequate dose coverage, acceptable dose homogeneity, and reliable deliverability. 


\section{Chapter 3}

\section{A four-dimensional computed}

\section{tomography study of a patient}

\section{immobilization system for breast}

\section{tomotherapy}

Accurate delivery of dose to the desired volume of tissue is critical to the outcome of radiotherapy. Setup errors, changes in the patient size because of either swelling or weight gain or loss, and intrafraction motion, primarily due to respiration, all work together to limit the accuracy with which dose can be delivered to the patient.

Usually in external beam radiation therapy this is dealt with by expanding the clinical target volume (CTV) by some predetermined margin to obtain a planning target volume 
(PTV) and then planning the treatment to encompass the entire PTV. A similar approach is used when treating breast cancer, where the CTV encompasses an entire breast; Report 50 of the International Commission on Radiation Units and Measurements ${ }^{19}$ (ICRU) recommends the use of 'flash'-an extension of the treatment beam beyond the surface of the patient--to account for these uncertainties. For conventional breast radiation therapy techniques using parallel-opposed tangential fields, extending the radiation field past the surface of the breast is relatively straightforward. However, with inverse planned treatments, extending the PTV outside the patient causes difficulties for the optimization algorithm because the relatively low density of the air makes it impossible to achieve the dose prescribed. ICRU Report 62 suggests adding an artificial, low-density bolus extending 5 $\mathrm{mm}$ past the PTV in all directions in order to facilitate optimization ${ }^{20}$.

Several studies have reported that as the amplitude of intrafraction motion increases, target dose coverage is compromised. Yue et al. have reported that for conventional conformal plans, patient motion may result in a significant increase or decrease to the minimum dose $\left(D_{\min }\right)$ delivered to the target ${ }^{21}$. The same study also reported that the maximum dose to the target $\left(D_{\max }\right)$ and dose to the heart were largely unaffected by the respiratory motion of the patient. With IMRT the intensity profile of the treatment beam varies continually while the patient is breathing, resulting in a dose distribution determined by a convolution of the time-dependent beam intensity and the position of the patient. A study published in 2003 by George $e t a l .{ }^{22}$ attempted to quantify this effect for breast IMRT treatment. They concluded that the predominant source of treatment degradation when using IMRT to treat 
breast cancer was the expanded PTV-CTV margins necessary to cover the target over the entire range of intrafraction motion. As the motion increased, so too did the size of the required PTV and consequently the dose to the heart and lungs. Dose heterogeneity and the difference between planned and expected dose were also shown to increase with respiratory motion.

By expanding the PTV volume enough to take into account the breathing motion and setup error, conventional breast radiation therapy techniques have achieved acceptable results without using any special immobilizing techniques. To take advantage of the high degree of precision afforded by the combination of IMRT and IGRT, tomotherapy requires additional means of immobilization to keep the patient correctly aligned during treatment. For breast cancer treatments at The Ottawa Hospital Cancer Centre, this takes the form of a thermoplastic shell extending from the top of the breast to mid-torso. However, even with immobilization, it is impossible to completely eliminate all patient motion; even if the surface of the patient is fixed, there will still be internal motion. For breast cancer treatment, breathing motion is the largest source of intrafraction motion.

Because tomotherapy has the ability to function as a CT scanner, patient misalignment can be corrected prior to beginning treatment. Were the body a rigid structure capable of perfectly maintaining its form from one day to the next, this would allow the patient to be aligned to an accuracy of better than $1 \mathrm{~mm}$ (the resolution of the software alignment tool) prior to each treatment. However, the body also undergoes slight changes which make the problem of alignment more complicated, since the setup can only compensate for linear 
shifts in the patient, not geometrical deformations. For the purposes of comparison we will also look at the daily shifts applied to patients at our center before undergoing treatment to get some sense of their magnitude relative to the intrafraction motion. It should be noted that in a conventional radiation therapy setup there has historically been no pre-delivery imaging and realignment, so one of the reasons for flash is to compensate for shifts in patient position, shifts that can be minimized when using an image guided modality like tomotherapy. In fact, with conventional radiation therapy, the uncertainty in position due to day-to-day variations in patient setup is typically much greater than the uncertainty due to respiratory motion ${ }^{23}$.

Most published research on patient motion has focused on reporting the dosimetric impact of patient motion during radiotherapy. Although the methodology varies, the typical approach to describing the consequences of patient motion has been to calculate the dose distribution that results when treating a moving patient using a plan generated from a free breathing scan of the patient ${ }^{21,22,24}$. Results are usually reported using dosimetric quantifiers: dose homogeneity, volume of the PTV receiving at least $95 \%$ of the prescribed dose $\left(V_{95}\right)$, etc. for the PTV, and metrics such as the volume receiving more than $20 \%$ of the prescribed dose $\left(V_{20}\right)$, average dose $\left(D_{\text {mean }}\right)$, and so on for the heart, lungs, and other organs at risk.

However, dosimetric data is one factor to be considered. Dosimetric parameters are sensitive to factors that have uncertain clinical significance. For example, the percentage of the total lung volume that receives more than a particular dose will be just as sensitive 
to the total lung volume as it is to subtle changes in the dose distribution. That is to say, a difference in the way that one patient breathes in comparison to another is as likely to affect the value of $V_{5}$ reported as a change to the way that radiation is delivered.

Dosimetric data also tends to bury other information that may be useful for comparing the likely clinical outcome of one treatment relative to another. For instance, various studies will report dose to the heart using a wide variety of disparate metrics: $D_{\max }, D_{\text {mean }}, V_{30 G y}$, $V_{5 G y}$, and $V_{D 95 \%}$ to name a few. The heart, obviously, is not a uniform mass of undifferentiated tissue, and certain sections of the heart are likely to exhibit greater sensitivity to long term radiation damage than others. If, for instance, a study shows that the $V_{5}$ parameter for the heart is unchanged by a particular modification of the procedure, it may still obscure any clinical consequences that may result if the bulk of the dose is absorbed in one case by the major coronary arteries and in the other by the cardiac muscle at the apex of the heart. One way around this may be to further subdivide the dose reporting into smaller structures that would allow for more specific description of dose deposition. In general however, a qualitative understanding of how the heart moves as the patient breathes may be beneficial in planning treatments to minimize dose to the heart.

\subsection{Breathing Motion}

Many studies have been done to measure the efficacy of various techniques for dealing with breathing motion during conventional radiotherapy. Certain techniques, including 
deep inspiration breath hold (DIBH) and active breathing control $(\mathrm{ABC})^{25,26}$, have been used to reduce the dose to the heart and lungs.

Where breathing motion is covered, it is usually described in terms of the maximum displacement of the breast surface—probably the most useful metric for determining the necessary CTV-PTV margins or the amount of flash required. Since most breast radiation therapy has been delivered using only gravity to immobilize the breast there has been no published work on the residual motion of patients immobilized with a thermoplastic shell.

The general mechanics of breathing itself are well understood. As would be expected, the number of degrees of freedom-voluntary vs. involuntary, breathing rate and depth, expansion of the ribcage, motion of the diaphragm, etc.-makes a quantitative description of breathing motion prohibitively complex. However, the intent here is to quantify the motion of a patient when immobilized in the position in which they will be treated. We hope to determine where motion can be successfully reduced to be clinically insignificant, and to anticipate the unavoidable motion that will be of clinical importance. Furthermore, an accurate understanding of the organ motion that occurs during treatment may be beneficial in weighing the tradeoffs inherent in treatment planning.

The goal of this portion of the study is to characterize the motion of the tissue within the vicinity of the treatment volume to understand its significance, anticipate the impact it is likely to have on the clinical outcome, and suggest ways of mitigating or avoiding any adverse consequences. Of primary interest is the motion of the surface of the breast, the lungs, and the heart. This was accomplished in this study using four-dimensional computed 
tomography.

\subsubsection{Breast Motion}

For the majority of patients who have undergone a lumpectomy, the CTV will include the entirety of the affected breast. Depending on the patient, the skin may be designated as either part of the target or an organ at risk. Excess dose to the skin can cause painful and disfiguring skin reactions, so where there is no reason to believe that the cancer has invaded the skin, dose to the skin should be minimized. In other cases, usually where the patient has undergone a mastectomy, the skin may be part of the target, and bolus may be necessary to achieve the desired dose near the surface.

Quantitatively describing the motion of the breast surface should help to determine whether there is any need to use flash when treating breast cancer with tomotherapy, set clear standards for the PTV-CTV margins (which may be different for the surface of the PTV next to the skin than for the surface next to the chest wall), and anticipate potential difficulties in delivering a uniform dose to volumes located at or near the surface of the breast.

\subsubsection{Lung Motion}

Being situated directly below the breasts makes the lungs, especially the ipsilateral lung, the primary organs at risk during breast cancer radiotherapy. Excess dose to the lungs can 
result in a variety of complications, most commonly pneumonitis or pulmonary fibrosis. For any given individual, breathing motion can exhibit a high degree of variability. When a person breathes, the lungs are expanded by a combination of diaphragm movement and ribcage expansion. It is probably not possible for these to be completely decoupled, though it is possible to change the degree to which each of these plays a role in expanding the lungs. It is hoped that by immobilizing the patient's chest during treatment most of the breathing will be regulated by the diaphragm, thereby leaving the chest wall as stationary as possible.

\subsubsection{Heart Motion}

Dose to the heart is of particular concern when treating breast cancer. Particularly when the cancer is in the left breast, the radiation dose absorbed by the heart may lead to cardiac complications at some later date ${ }^{27,28}$.

Because the heart sits between the lungs and directly on top of the diaphragm, it moves as the patient breathes. One possible advantage of using tomotherapy is the potential for treating the inner mammary lymph chain while avoiding the heart, something not possible using opposed tangential fields. Irradiation of the inner mammary lymph chain is somewhat controversial. Two studies published in the New England Journal of Medicine in 1997 showed a clear survival benefit for node-positive pre-menopausal women receiving radiation therapy in addition to chemotherapy ${ }^{4,29}$. In both clinical trials the radiation therapy included irradiation of the internal mammary chain, but because the IMC was included 
in the PTV for all patients, it is impossible to draw any conclusions from either of these trials as to whether including or excluding the IMC is in itself beneficial. However, it is reasonable to suggest that the irradiation of all of the lymph nodes will be of clinical benefit, provided that including the IMC in the target does not result in excess damage to the heart that ultimately counters any benefits of including the IMC. Clinical trials to test this hypothesis are ongoing.

\subsection{Materials and Method}

\subsubsection{Patients}

The patients included in this study were part of a larger study of the feasibility of postoperative locoregional radiation therapy using tomotherapy for the management of stage IIb-III breast cancer. All patients had been diagnosed with adenocarcinoma with no evidence of metastatic disease. All patients had undergone either mastectomy or breastconserving surgery and had exhibited high risk pathological features at the time of surgery, defined as involvement of the locoregional lymph nodes. The study included women with both left and right sided disease. Of the eight patients analyzed, three had undergone breast conserving surgery, one on the left and two on the right, and five had undergone mastectomy, three on the right, one on the left, and one on both sides. 


\subsubsection{Immobilization and Breath Coaching}

The patients were positioned on a MEDTEC breastboard (CIVCO, Kalona, IA), supine, with both arms abducted above their head. Their breasts were immobilized using a thermoplastic shell. For patients who had undergone a lumpectomy, the ipsilateral breast was pushed up to the front of the chest and the contralateral breast allowed to fall to the side of the torso. For those patients who had undergone a mastectomy, bolus was placed over top of the thermoplastic shell over the surgical bed of the mastectomy to make it easier to control dose to the skin, which was included in the target volume for those patients.

\subsubsection{4-Dimensional Computed Tomography}

On an ordinary $\mathrm{CT}$ scan, the motion associated with breathing will be blurred by the finite time required to perform the scan. $4 \mathrm{D} \mathrm{CT}$ scanning allows some periodic motion to be resolved, provided that the periodicity of the motion can be accurately measured by some surrogate. Possible surrogates include the actual breathing of the patient, measured by a spirometer, or external patient motion, monitored by observation of fiducials with a camera system or physically measuring motion of the patient's abdomen or chest wall ${ }^{30}$.

During this study the intent was to keep the chest wall of the patient as stationary as possible. Motion of the abdomen, however, was taken to be a valid surrogate for tracking the patient's breathing cycle. An elastic belt with an accordion shaped air bellows connected to a pressure transducer (Philips Medical Systems, Cleveland, $\mathrm{OH}$ ) was placed 
around the abdomen of the patient to monitor its circumference and the data fed into the scanning software. The time it took the patient to go through a complete cycle of inhalation and exhalation was divided into ten equal periods, or breath phases. For the 4D CT scan the patient was moved through the bore of the CT scanner in axial mode. At each position the scanner acquired sixteen slices simultaneously over several full breathing cycles in order to collect enough data to reconstruct a discrete image of each slice for each phase. (This also required the patient to remain as motionless as possible for a considerably longer period than for a regular helical CT scan.) The data was then sorted by breath phase prior to reconstruction, resulting in a full three dimensional CT reconstruction of the patient for each breath phase.

For this study, the patients underwent both a conventional, free-breathing helical CT scan, and a 4DCT scan. For both the slice thickness was $3.0 \mathrm{~mm}$. The CT resolution in the transverse plane was $0.97 \mathrm{~mm}$.

Patients were asked to breathe normally for this study; maximum inhalation and exhalation are used below in reference to the patient's normal breathing cycle, not the limits of which the patient is capable by consciously inhaling or exhaling.

\subsubsection{Contouring and Measurements}

Following acquisition, the skin and lungs were contoured for each phase using the FocalSim (CMS Inc., St. Louis, MI) auto-contouring tools. Aside from the efficiency advantage, the auto-contouring tool removes at least one source of subjectivity in determining where 
to draw the contours. The auto-contour tool works by searching for boundaries between different areas of the CT image and drawing a closed loop around that boundary. The contrast of the image can be adjusted using the level and window settings which determine the image value (in CT number or Hounsfield units) corresponding to $50 \%$ grey, and the range of CT number values over which the image goes from black to white. The software uses the gray values of the displayed image to determine the location of tissue boundaries; thus, by adjusting the contrast of the image the location of the contours drawn by the auto-contouring tool can be altered. The lungs were contoured using the AutoLung setting (Level: -850, Window: 1300), and the skin using the AutoSkin setting (Level: 400, Window: 1200). In the cases of patients with bolus on top of the immobilization device, the level and window settings were sometimes adjusted to 500 and 1200 respectively to avoid contouring the bolus rather than the skin. In some cases, particularly for those patients with bolus placed over the shell, it was necessary to manually touch up the contours where the immobilization mask and bolus was incorrectly included in the contour by the auto-contouring tools.

Once the CTs had been contoured, the challenge was to quantify the observed motion and report the results in a consistent and meaningful manner. The first step for each patient was to determine the breath phases corresponding to maximum inhalation and maximum exhalation. The phase in which the diaphragm position was highest was selected as the phase of maximum exhalation. The maximum displacement of the inferior surface of the lung in the superior-inferior direction for each patient is reported in Table 3.1. 
Figure 3.1 shows the lung and skin contours at the mid-breast level for one of the patients. The contours for the phases corresponding to minimum and maximum inhalation are overlaid on the free breathing CT of the patient.

Having determined the limiting phases, the next step was to measure the anatomical displacement between the two phases. To simplify reporting, we chose to report motion for three transverse slices for each patient: one slice at mid-breast and slices $51 \mathrm{~mm}$ superior and inferior to the mid-breast. The mid-breast was marked on each CT scan by a radioopaque marker placed on centre of the patient's chest, approximately level with the center of the intended target volume. Skin motion was defined as the displacement of the front surface of the breast in the anterior-posterior direction (see Figure 3.1).

In this study we also report the motion of the front wall of the right and left lung in the anterior-posterior direction, a metric essentially equivalent to the motion of the patient's chest wall. Measurements were made on the most anteriorly located segment of the lung contour.

As the diaphragm rises, the heart is pushed upwards and somewhat into the medial wall of the left lung. The value we measured and report here shows the displacement of the wall of the left lung in the left-right direction corresponding to an expansion of the organ at risk in the axial plane. Heart motion was measured for both the mid-breast slice and the slice $51 \mathrm{~mm}$ above the mid-breast. For most patients, the transverse slice $51 \mathrm{~mm}$ below the mid-breast intersects the bottom of the heart during maximum inhalation and no heart motion data was measured for that slice. 


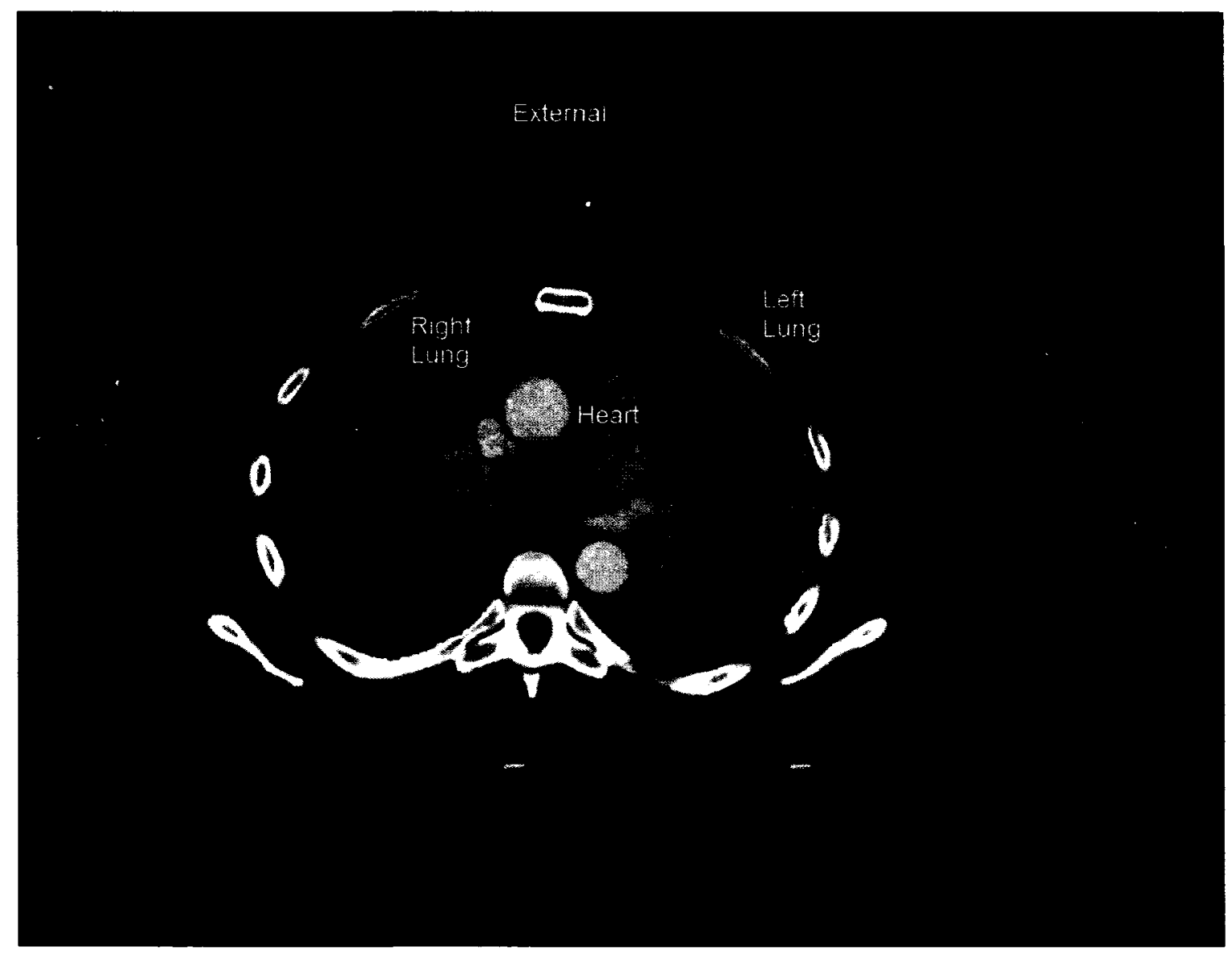

Figure 3.1: Transverse view of patient at mid-breast, looking towards the head, showing lung and skin contours for patient 3 at mid-breast. Contours at maximum inhalation are shown in blue, maximum exhalation in red. Contours are overlaid on free breathing CT. Markers indicate where displacement was measured for the external surface, lungs and heart. 
The internal mammary lymph chain follows the inner mammary vessels, located just behind the chest wall to the left and right of the sternum. Since the lymph nodes themselves are not visible on a CT scan, the volume of the IMC is defined by an expansion around the vessels which are discernable on a CT scan (though injection of a contrast agent may be necessary to make them clearly visible). We assume the motion of the chest wall near the sternum to be a reasonable proxy for the motion of the inner mammary chain.

The diaphragm motion was measured primarily as a means of determining the phases corresponding to maximum inhalation and exhalation for each patient. Since the motion of a large, deformable surface cannot be accurately captured by a single measurement, the values reported here represent the approximate maximum displacement of the bottom of each lung near the center of the patient in the superior-inferior direction.

All measurements were made by using the FocalSim software 'ruler' tool to measure the separation between the contours in the region of maximum displacement. The arrows on Figure 3.1 indicate the approximate locations of the reported measurements. We estimate the overall uncertainty of the measurements made in the transverse plane to be on the order of about 1-2 mm, taking into account the limited resolution of the scan itself $(1 \mathrm{~mm})$, the resolution of the software tools used to make the measurements $(1 \mathrm{~mm})$, and the nonuniformity of the motion of the contours being measured. Diaphragm motion was measured in the coronal plane, and has a larger uncertainty of about $3 \mathrm{~mm}$ due to the thickness of the CT slices. 


\subsubsection{Shift data analysis}

Prior to delivery of each dose fraction to a patient, the patient is first scanned using the tomotherapy system in MVCT mode. This scan is then registered to the kVCT scan used to create the treatment plan. Any adjustments in the X (left-right), $\mathrm{Y}$ (superior-inferior) and $\mathrm{Z}$ (anterior-posterior) directions necessary to align the patient to her original position are then applied to the patient before delivering dose. For each of ten breast cancer patients treated at The Ottawa Hospital Cancer Center, a group which included all of the patients analyzed by the 4D CT scans as well as two others for whom 4DCT data was unavailable, we are reporting the total shift applied to each patient in each direction prior to treatment, averaged over the full course of treatment ( 25 fractions). To observe how the necessary shifts vary from day to day, we also report the standard deviation for each data set. These two values are taken to correspond to the systematic and random uncertainty in the daily alignment of the patient following the initial setup, but prior to registration.

\subsection{Results and Analysis}

\subsubsection{D CT study}

Table 3.1 shows the displacement of the skin, lung and heart contours in the plane at the mid-breast level. Displacement in the plane $51 \mathrm{~mm}$ superior to the mid-breast is shown in Table 3.2. 
Table 3.1: Maximum displacement during free-breathing at the mid-breast level for a patient immobilized with a thermoplastic shell, in mm. (-) indicates that the CT scan showed no clear evidence of motion during the breathing cycle. N/A indicates where data could not be measured for a particular patient.

\begin{tabular}{ccccccc}
\hline \hline Patient & Breast Surface & Left Lung & Right Lung & Heart & IMC & Diaphragm (Rt/Lt) \\
\hline 1 & - & - & 2 & 11 & 3 & $23 / 6$ \\
2 & - & N/A & - & 13 & 2 & $17 / 12$ \\
3 & 4 & 2 & 3 & 12 & N/A & $16 / 19$ \\
4 & 2 & 3 & 3 & 5 & 2 & $7 / 10$ \\
5 & - & - & - & 6 & N/A & $18 / 11$ \\
6 & 2 & - & - & 7 & N/A & $18 / 16$ \\
7 & - & - & - & 12 & - & $17 / 11$ \\
8 & - & - & - & 8 & - & $23 / 18$ \\
\hline \hline
\end{tabular}

The motion observed in the slice $51 \mathrm{~mm}$ inferior to the mid-breast was in all cases less than or equal to our estimated margin of error $(2 \mathrm{~mm})$. It can be seen from the data that the thermoplastic shell is very effective at immobilizing the patient during treatment. For most patients, the external surface is displaced by less than $2 \mathrm{~mm}$ over the entire breathing cycle, with a maximum displacement of $4 \mathrm{~mm}$. The displacement of the external surface $51 \mathrm{~mm}$ superior and $51 \mathrm{~mm}$ inferior to the mid-breast was in every case less than the displacement at mid-breast. In none of the patients did the excursion of the front surface of the lung/chest 
Table 3.2: Displacement $51 \mathrm{~mm}$ superior to mid-breast level, in $\mathrm{mm}$. (-) indicates that the CT scan showed no clear evidence of motion during the breathing cycle.

\begin{tabular}{ccccc}
\hline \hline Patient & External & Left Lung & Right Lung & Heart \\
\hline 1 & - & - & 2 & 5 \\
2 & - & - & 3 & 6 \\
3 & 3 & 5 & 5 & 7 \\
4 & - & 2 & 2 & 4 \\
5 & 2 & - & - & 3 \\
6 & - & - & - & 3 \\
7 & - & - & - & 7 \\
8 & - & - & - & 4 \\
\hline \hline
\end{tabular}

wall at mid-breast exceed $3 \mathrm{~mm}$. A single patient exhibited displacement of the chest wall of approximately $5 \mathrm{~mm}$ in the slice $51 \mathrm{~mm}$ superior to the mid-breast. In none of the other patients was there evidence of displacement significantly exceeding the $2 \mathrm{~mm}$ in the slices superior and inferior to the mid-breast. Looking at a transverse view of the patient, the heart appears to be moving significantly to the left, though this is primarily because the heart wall is angled down and to the left and rises with the diaphragm as the patient breathes.

The quantitative data reported for the motion of the diaphragm confirms that the presence of the thermoplastic immobilization shell is forcing the patients to breathe almost entirely with the diaphragm while keeping the chest wall stationary, as intended. 


\subsubsection{Pre-treatment Shift Data}

Table 3.3 shows the average amount by which each of ten patients was shifted in the left-right (LR), anterior-posterior (AP), and superior-inferior (SI) directions after the daily alignment $\mathrm{CT}$. The average shift for each patient is taken to be the systematic (preparation) error for that patient, and the standard deviation of the shift required is taken to be the random setup error for that patient.

The required average shift in the AP direction was positive in every case, averaging $7.6 \mathrm{~mm}$. The required shift was relatively consistent from day to day, with the standard deviation of the shift falling in the range of 1-3 mm for all but one of the patients.

In the LR direction, the average shift required was $-0.3 \mathrm{~mm}$, consistent with there being no systematic misalignment of the patient in the LR direction. The standard deviation of the LR shift was between 2 and $4 \mathrm{~mm}$ with only one exception.

In both the AP and LR directions the same patient (number 7) exhibited the highest standard deviation of the pre-treatment shift required.

In the SI direction, the average magnitude of the required shift was $7.8 \mathrm{~mm}$, with patients requiring shifts averaging between $-17 \mathrm{~mm}$ and $+15 \mathrm{~mm}$. The average shift required was $-2.7 \mathrm{~mm}$. The standard deviation of the daily shift was significantly greater for all patients in the SI direction, ranging from just over $4 \mathrm{~mm}$ in one patient to over $13 \mathrm{~mm}$ in another.

To get a better sense of the overall uncertainty in patient immobilization and setup, we 
Table 3.3: Average and standard deviation of daily shifts required in the left-right (LR), anterior-posterior (AP), and superior-inferior (SI) directions after the alignment MVCT scan, in $\mathrm{mm}$. Also shown are the overall systematic $(\Sigma)$, and random $(\sigma)$ errors.

\begin{tabular}{ccccccc}
\hline \hline & \multicolumn{2}{c}{ LR } & \multicolumn{3}{c}{ SI } & \multicolumn{2}{c}{ AP } \\
Patient & average & $\sigma$ & average & $\sigma$ & average & $\sigma$ \\
\hline 1 & 1.9 & 2.6 & 1.3 & 4.5 & 3.3 & 1.9 \\
2 & -3.6 & 2.1 & -17.2 & 13.5 & 4.8 & 1.9 \\
3 & 3.8 & 3.6 & -3.2 & 7.3 & 6.3 & 2.2 \\
4 & -0.1 & 2.3 & -6.4 & 5.6 & 6.9 & 2.3 \\
5 & -3.5 & 3.1 & 3.0 & 5.9 & 10.1 & 1.7 \\
6 & 1.6 & 3.0 & -14.0 & 8.2 & 5.4 & 1.4 \\
7 & -3.8 & 5.5 & -11.6 & 8.8 & 2.1 & 3.8 \\
8 & 1.5 & 2.8 & 1.4 & 6.0 & 9.8 & 2.9 \\
9 & 0.4 & 2.4 & 4.4 & 6.2 & 11.4 & 1.7 \\
10 & -1.4 & 2.6 & 15.2 & 8.0 & 15.5 & 2.3 \\
\hline & $\Sigma_{L R}=2.7$ & $\sigma_{L R}=3.1$ & $\Sigma_{S I}=9.8$ & $\sigma_{S I}=7.8$ & $\Sigma_{A P}=4.1$ & $\sigma_{A P}=2.3$ \\
\hline \hline
\end{tabular}


calculated overall systematic error $(\Sigma)$ as the standard deviation of the average shifts for all patients, and overall random error $(\sigma)$ as the root mean square of the random shift errors for all patients. This resulted in systematic setup errors in the LR, SI, and AP directions of $2.7 \mathrm{~mm}, 9.8 \mathrm{~mm}$, and $4.1 \mathrm{~mm}$ respectively. Random errors were $3.1 \mathrm{~mm}, 7.8 \mathrm{~mm}$, and $2.3 \mathrm{~mm}$ respectively. The greater uncertainty in the superior-inferior setup position may be partially attributable to the lower resolution in the axial dimension $(3.0 \mathrm{~mm})$ relative to the transverse resolution $(1.9 \mathrm{~mm})$, though this fails to fully account for the difference.

Neither the thermoplastic shell, nor the surface of the patient with which it is in contact are perfectly rigid. Moreover, the muscles and bony anatomy used to register the daily CT scan relative to the planning scan are themselves subject to small temporal variations in size and position. The systematic and random error in the patient position clearly suggests that expansion of the treatment beam would be necessary to guarantee coverage if the patient position were not re-registered to the original CT scan. The CTV-PTV margin necessary to guarantee a minimum dose of $95 \%$ to $90 \%$ of patients, as per van Herk et al. ${ }^{31}$, is given by:

$$
m_{P T V}=2.5 \Sigma+0.7 \sigma^{\prime}
$$

where $\Sigma$ is the systematic error and $\sigma^{\prime}$ is the combined random error ${ }^{1}$. Using the errors reported here, this results in a CTV-PTV margin of $33 \mathrm{~mm}$.

However, since the patient position is adjusted based on the MVCT imaging prior to beginning treatments, the actual uncertainty in position during treatment will be much smaller.

\footnotetext{
${ }^{1}$ This oversimplifies somewhat, and doesn't really translate directly to tomotherapy, since it includes assumptions about the beam penumbra more applicable to conventional radiation therapy.
} 
Daily registration corrects for both systematic and random error in patient setup. Measurement of the true residual setup error during treatment would require that the patient be rescanned after registration, possibly following treatment. This approach has been used to measure the patient setup fidelity for prostate, head and neck, and glioblastoma treatments ${ }^{32}$ using tomotherapy and has been reported to achieve post treatment misalignments of less than $1 \mathrm{~mm}$ in all axes for all three treatment sites, but no published results were found for tomotherapy breast treatment. An investigation of a non-tomotherapy setup which used cone-beam CT to align patients for accelerated partial breast irradiation found residual systematic errors of $0.7-0.8 \mathrm{~mm}$ and residual random errors of $1.5-1.6 \mathrm{~mm}^{33}$.

\subsection{Conclusions}

Overall displacement of the tissue at the boundaries of the target volume, namely the chest wall and the surface of the breast, was limited to less than $2 \mathrm{~mm}$ in the majority of the patients studied. In only one patient was the motion as much as $5 \mathrm{~mm}$, and even for that patient only the chest wall $51 \mathrm{~mm}$ superior to the mid-line exhibited that much motion. Therefore, the magnitude of the motion is on approximately the same scale as the voxel size used by tomotherapy in calculating the dose. The magnitude of the breathing motion is comparable to the uncertainty in the position of the patient due to day-to-day variations in set up, which we estimate to no more than $1-2 \mathrm{~mm}$ as per White et al. ${ }^{33}$. Combining these two independent uncertainties, the overall uncertainty in the position of the target is 
still less than the width of a single beamlet $(6.25 \mathrm{~mm}$ at central axis), suggesting that it is probably unnecessary to introduce the use of flash in tomotherapy when the breast is immobilized as described. At the tissue boundaries where flash might hypothetically be introduced, it is likely that the beamlets used will already, because of the beamlet width and calculation voxel size, overshoot the target slightly by a sufficient margin to account for any breathing motion. 


\section{Chapter 4}

\section{Dosimetric Consequences of PTV Depth}

\subsection{PTV Depth and Buildup Region}

Treating tissue near the surface of the patient presents certain challenges not encountered when treating deeper tissues. When a beam of $\mathrm{X}$-ray photons passes through matter it interacts with the material by transferring energy to electrons by various mechanisms: photoelectric effect, Compton scattering, and pair (or triplet) production. The degree to which each of these phenomena contributes to the transfer of energy from the photon beam to electrons depends on the energy of the photons involved and the material with which they are interacting. The energetic electrons set in motion then deposit their energy gradually through a series of collisions and electrostatic interactions with other particles, primarily other electrons. Thus, energy deposition occurs not at the site of the initial photon interaction, but is instead 'smeared out' along the path of the electron set in motion by that 
interaction. As the electron slows down and deposits its energy it will undergo repeated scattering and follow a rather convoluted path, knocking other electrons loose in the process. Since, on average, most electrons will be scattering in the forward direction, energy deposition will occur mostly "downstream" from the original photon interaction. Furthermore, because the electrons are depositing energy as they go, they will have a finite range that depends on their initial energy and the rate at which they deposit energy (defined as the stopping power of the material in which they are traveling).

The total fluence of electrons passing through a given point will therefore depend mostly on the photon interactions that occur "upstream" of that point. The total number of photon interactions depends heavily on the electron density of the material with which the photon beam is interacting. When a beam of photons passes through air, relatively few electrons are set in motion compared to the number of photons which would be set in motion by the same beam passing through water or tissue which is about a thousand times more dense.

If we consider a beam of photons passing through a volume of air and impinging on a block of tissue (or any other similarly dense matter), the total fluence of electrons at the surface of the tissue will be relatively small. Just inside the tissue, the fluence will be much higher, as, in addition to a few electrons from the photon interactions in air, there are now many electrons set in motion by photon interactions in the tissue. The total electron flux increases with increasing depth until we reach a depth equal to the maximum range of the electrons, at which point the total electron fluence will begin to fall off as the photon beam 
is attenuated by the tissue.

The dose deposited at any point can be calculated from the electron flux at that point, $\Phi$, and the collisional mass stopping power of the material, $(S / \rho)_{c o l}$, where

$$
D=\Phi\left(\frac{S}{\rho}\right)_{c o l} . \quad[G y]=\left[\frac{J}{k g}\right]
$$

More rigorously, the electron fluence will be multi-energetic and mass stopping power depends on the electron energy, thus:

$$
D=\int_{0}^{E_{\max }} \frac{d \Phi}{d E}\left(\frac{S(E)}{\rho}\right)_{c o l} d E . \quad[G y]
$$

The net effect of all of this is that the volume just below the surface of a patient will actually receive considerably less dose than the tissue a few millimeters deeper. This effect, known as 'skin sparing,' is often desirable when treating patients, since skin reactions from radiation therapy can be extremely painful. However, in other situations, particularly when the volume to be treated is located right below the skin, or even includes the skin itself, skin sparing can make it difficult to achieve the desired dose without overdosing the patient below the surface.

Using tomotherapy to treat patients introduces a further complication, since tomotherapy may often use beams that instead of impinging directly on the skin intersect the surface of the patient at a shallow angle. This is particularly relevant to the treatment of breast cancer, which uses beams that are frequently tangential to part of the breast surface to avoid delivering dose to the organs at risk within the chest. Studies have shown that superficial 
dose is substantially higher from oblique tomotherapy beams than from beams at normal incidence ${ }^{16}$.

In a paper published in 2007, Ramsey et al. ${ }^{34}$ used an anthropomorphic phantom to look at the superficial doses calculated and delivered in head and neck treatments. This study reported that the PTV received a maximum of $109 \%$ and a minimum of $66 \%$ of the prescribed dose when the PTV was extended to the surface of the phantom, and that uniformity improved as the margin between the PTV and the surface of the phantom was increased. The same study also reported that the tomotherapy software overestimated superficial dose by about $8.9 \pm 3.2 \%$ compared to that measured using TLDs.

Cherpak et al. compared the dose calculated by the tomotherapy software to the dose measured by TLDs and MOSFETs for head and neck patients ${ }^{35}$. They reported an average absolute disagreement between the treatment plan and the surface dose, as measured using MOSFETs, of $12.2 \pm 7.5 \%$, most of which was attributed to partial volume effects at the airsurface interface. Whether the treatment planning system overestimated or underestimated dose depended on the point in question.

Though the issues involved are similar, the results of these studies probably cannot be applied directly to the treatment of breast cancer. Even though both scenarios involve the treatment of PTV located directly adjacent to the skin, when treating breast cancer the PTV is much larger than for head and neck cancers and the geometry is substantially different. For breast cancer treatment, most of the dose will be delivered by beams that are tangential to the patient and far from the center of the gantry. The setup of the head and neck study 
permitted beams from nearly all angles. Because the heart and lungs are the primary organs at risk, none of the beams used to treat breast cancer enter from below the breast.

When a PTV is contoured and assigned a dose, the treatment planning software will usually attempt to create a plan that delivers a uniform dose to the entire volume. However, delivering uniform dose to an arbitrary region is especially difficult for volumes near the surface of the patient, as described previously in the section dealing with the buildup region. The first section of this work will attempt to describe the interaction between the location of the PTV, in particular its proximity to the surface of the patient, and the dose uniformity of the resulting treatment plan.

Calculating and predicting dose at and near the surface of a patient or phantom without a full Monte Carlo simulation is a challenge. The TomoTherapy Hi-Art software uses a superposition algorithm (described briefly in section 2.2 ) that calculates dose by convolving the calculated TERMA with pre-calculated dose distribution kernels. In regions of high electronic disequilibrium, the scaled dose distribution kernel will not accurately reflect the true energy deposition. This happens because the dose to any particular voxel is deposited by electrons that have been scattered into that voxel from an adjacent one. Thus, dose to a particular voxel will depend on the density of those surrounding it, even if those voxels do not lie between the point in question and the TERMA point. The kernel scaling algorithm, however, looks only at the radiological distance between the TERMA point and the dose deposition point, and ignores the influence of the adjacent tissue. Consequently, the calculated dose for tissue near a tissue-air interface will be higher than the actual dose. 
Having established the expected behavior of the treatment planning software in response to varying the depth of a PTV within a phantom that has been carefully aligned, it will also be advantageous to examine how sensitive a particular plan may be to misalignment. Even with well designed immobilization techniques there will still be some residual motion and misalignment when treating an actual patient instead of a phantom. This could be due to inter-fraction effects, such as the patient losing or gaining weight, set up error, or intrafraction motion, as covered in chapter 3 .

Predicting surface dose with any accuracy is difficult. The doses calculated are necessarily averaged over an entire voxel, and the voxels containing the surface of the phantom contain both air and phantom, and are unlikely to accurately represent the true dose to the surface. Nevertheless, to determine whether it is possible to make any meaningful surface dose predictions with tomotherapy, we will also measure surface dose and compare it to the dose predicted by software for the voxels adjacent to the surface.

\subsection{Materials and Method}

\subsubsection{EDR2 Film}

Most dose measurements were made using Kodak EDR2 film. EDR2 film is commonly used for IMRT dose verification where steep dose gradients and complex dose distributions require high spacial resolution. The response of EDR2 is nearly linear with dose over a range extending from $0-350 \mathrm{cGy}^{36}$. With careful calibration, it can be used to obtain IMRT 
dose measurements in agreement with ion chamber point measurements to an accuracy of about $5 \%{ }^{37}$.

The relationship between optical density and dose for EDR2 film exhibits some variance, particularly from one batch of film to the next. One study reported variations in optical density from $7-15 \%$ of the average optical density over an eighteen month time period $^{37}$. Therefore, daily film calibration was performed prior to making measurements.

The film calibration method used is described in a paper by Yan et al. ${ }^{38}$. The calibration program consists of an MLC control file that delivers a stepped dose intensity pattern with a stationary gantry (Figure 4.1). The resulting dose profile is shown in Figure 4.2. The tomotherapy beam has a cone-shaped profile. Therefore, to create the flat steps needed for an effective film calibration, the MLC control file is designed to both compensate for the non-uniform beam profile and create a series of flat dose steps varying from approximately $45 \mathrm{cGy}$ in the centre to approximately $270 \mathrm{cGy}$ at the outer edge.

For film calibration, the film was placed inside a rectangular solid water phantom at a depth of $6 \mathrm{~cm}$. The film plane was vertically aligned with the isocenter of the tomotherapy gantry.

To measure the relative dose to each step of the intensity pattern, the calibration treatment plan was delivered eleven times to eleven different films, each time with an ion chamber centered under a different step of the intensity pattern. These measurements were used to determine the relative dose relationship between each of the eleven steps in the intensity pattern. Assuming that the output of the tomotherapy linac remains constant during a single 

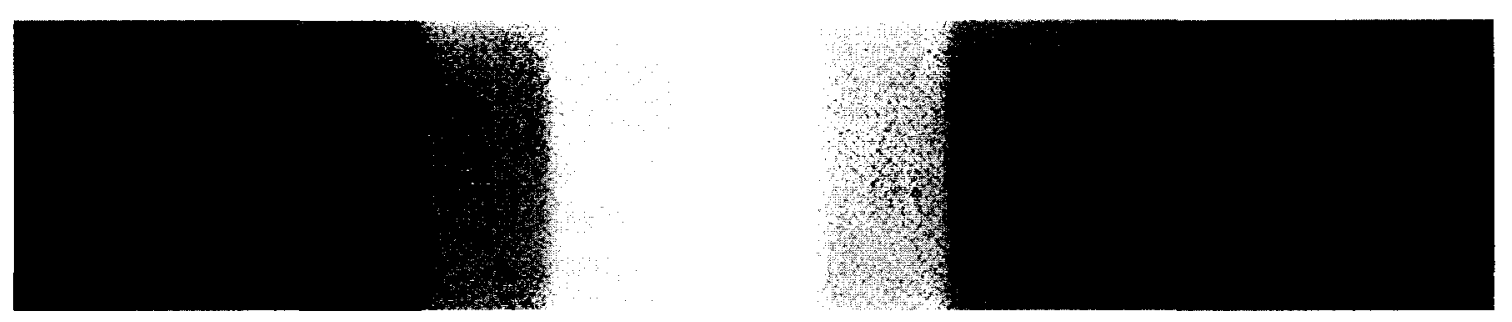

Figure 4.1: Calibration film.

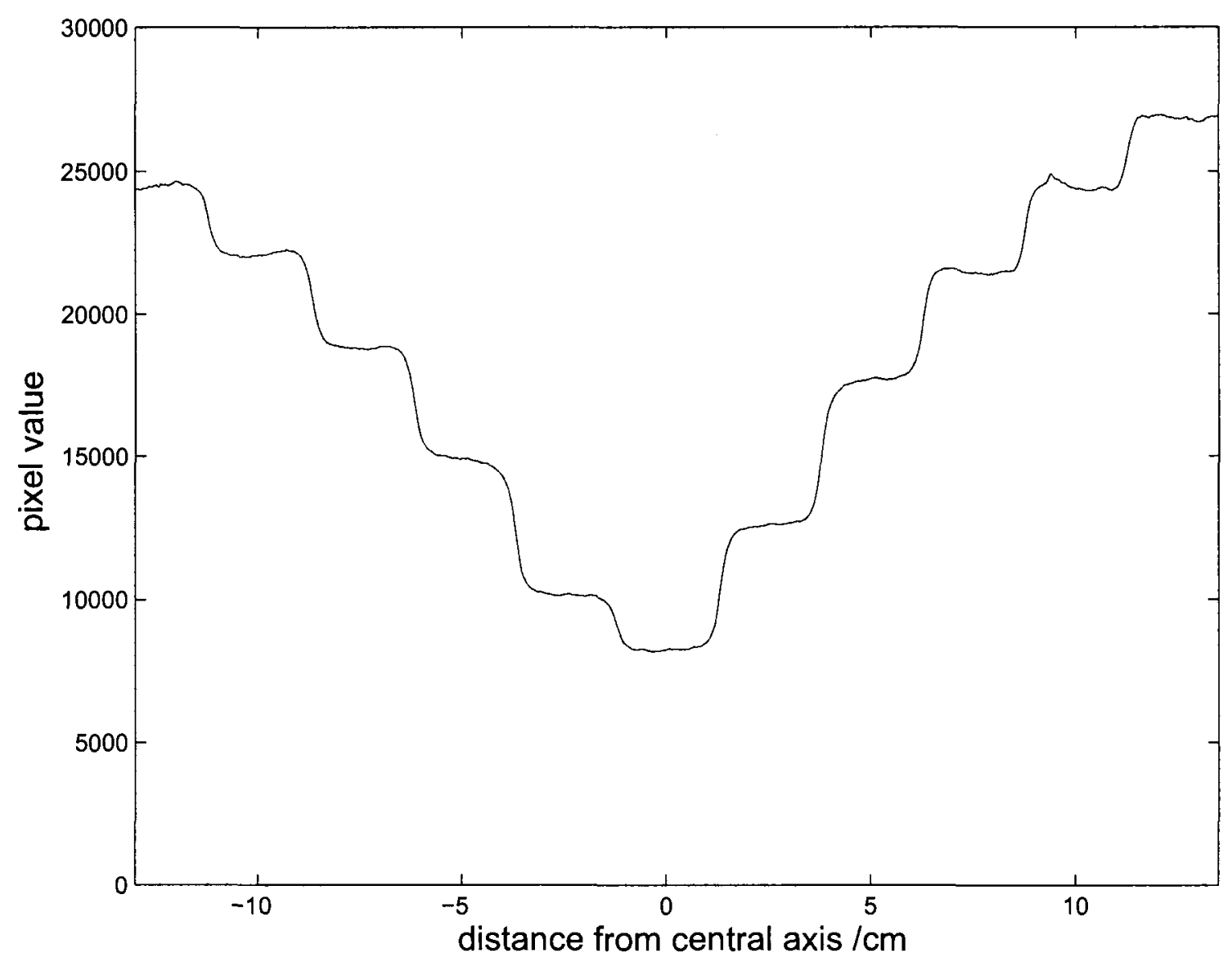

Figure 4.2: Optical density profile of calibration film along the $\mathrm{X}$ axis. Pixel values are in arbitrary scanner units. 
treatment (a valid assumption according to Fenwick et al. ${ }^{39}$ ), the relative dose in each step will also remain the same each time the treatment is delivered, since the dose to each step is controlled by the same MLC control file. Thus for each subsequent film calibration it is necessary only to measure the dose under the central step using an ion chamber and scale as required.

An Exradin A1SL ion chamber was used to make an accurate measurement of the absolute dose for reference purposes. In every case, the ion chamber was positioned $1 \mathrm{~cm}$ below the film plane. The dose to the ion chamber was calculated using the dose calibration parameters determined when the chamber was last cross-calibrated with an externally calibrated chamber at The Ottawa Hospital Cancer Center. To account for the difference between the depth of the film and the depth of the ion chamber, the dose measured by the ion chamber was scaled using the percentage depth-dose (PDD) curve measured in water for the tomotherapy beam.

For each day that measurements were taken, the films used for calibration and those used for measurement were all confirmed to have identical lot numbers.

It has also been reported that the relationship between optical density and dose depends on the time delay between exposure and development ${ }^{40}$. EDR2 film was reported to underrespond by about $4 \%-6 \%$ when developed 2 minutes after exposure, and by about $1 \%$ when developed 1 hour later. The optical density was reported to be stable for delays greater than 3 hours. To minimize any uncertainties arising from processing delay the film was exposed in the evening and developed the following day. 


\subsubsection{Gafchromic Film}

Surface dose measurements were made using Gafchromic EBT radiochromic film. The active layer in Gafchromic film forms a blue polymer when exposed to radiation. It has the advantage of being easy to work with since it is self-developing and is relatively insensitive to room light and thus does not need to be kept in a lightproof envelope. Thus, a piece of film may be cut to size and taped to the surface of the phantom to measure surface dose. Following exposure the Gafchromic film was scanned using an Epson V700 scanner (Seiko Epson Corp., Japan). The dose was calculated by using a curve correlating the average value of the blue channel of the resulting TIFF file to dose, calibrated for the particular lot of Gafchromic film being used. Because Gafchromic film also exhibits a time dependent optical density response ${ }^{41}$, the film was scanned 48 hours after exposure to allow the film to stabilize.

\subsubsection{Treatment Planning}

All tests were done using a cylindrical solid water phantom (TomoTherapy Inc., Madison, WI) with a radius of $15 \mathrm{~cm}$ and a length of $18 \mathrm{~cm}$. The curved surface of the phantom was used to approximate the surface of a breast and the remainder of the phantom to represent the organs at risk behind the breast (the heart and lungs). To make setup easier by keeping the film plane horizontal, the breast was drawn beside, rather than on top of the organ at risk, effectively rotating the phantom anatomy $90^{\circ}$ relative to the supine patient it is intended to 
represent. The target region was defined to include the volume between the surface of the phantom and the $\mathrm{Y}-\mathrm{Z}$ plane located at a depth of $10 \mathrm{~cm}$ (measured from the surface) as shown in Figure 4.3. The target had a length of $12 \mathrm{~cm}$ in the axial $(\mathrm{Y})$ direction and was centered along the length of the phantom. To investigate the relationship between the PTV depth and dose distribution, a total of six PTVs were created with skin-to-PTV depths of 0 $\mathrm{mm}, 2 \mathrm{~mm}, 4 \mathrm{~mm}, 6 \mathrm{~mm}, 8 \mathrm{~mm}$, and $10 \mathrm{~mm}$. In every case, the interior surface of the PTV remained the same. The volume of the phantom behind the segment defined as the target, and extending the entire length of the phantom, was defined as an organ at risk (OAR) to force tangential beam delivery as would be expected in clinical planning. The OAR was extended the entire length of the phantom. The CT data set was contoured in FocalSim (CMS, Inc., St. Louis, MO) and then exported to the tomotherapy planning system.

For each experiment, plans were generated using a jaw width of $2.5 \mathrm{~cm}$, a pitch of 0.300 , and the fine dose calculation grid $(1.9 \mathrm{~mm} \times 1.9 \mathrm{~mm} \times 3 \mathrm{~mm}$ (in the axial direction)). The PTV was assigned a target dose of 50 Gy to $97 \%$ of the volume. The OAR was assigned a maximum dose of 30 Gy with the requirement that no more than $20 \%$ of the volume receive more than $20 \mathrm{~Gy}$. A consistent penalty weighting of 10 was assigned for failing to meet any of the specified criteria. These optimization parameters are summarized in Table 4.1.

\subsubsection{CT data to relative mass density conversion}

The tomotherapy planning system uses a convolution/superposition algorithm to simulate the interaction of each beamlet with the material inside the irradiated volume. This volume 


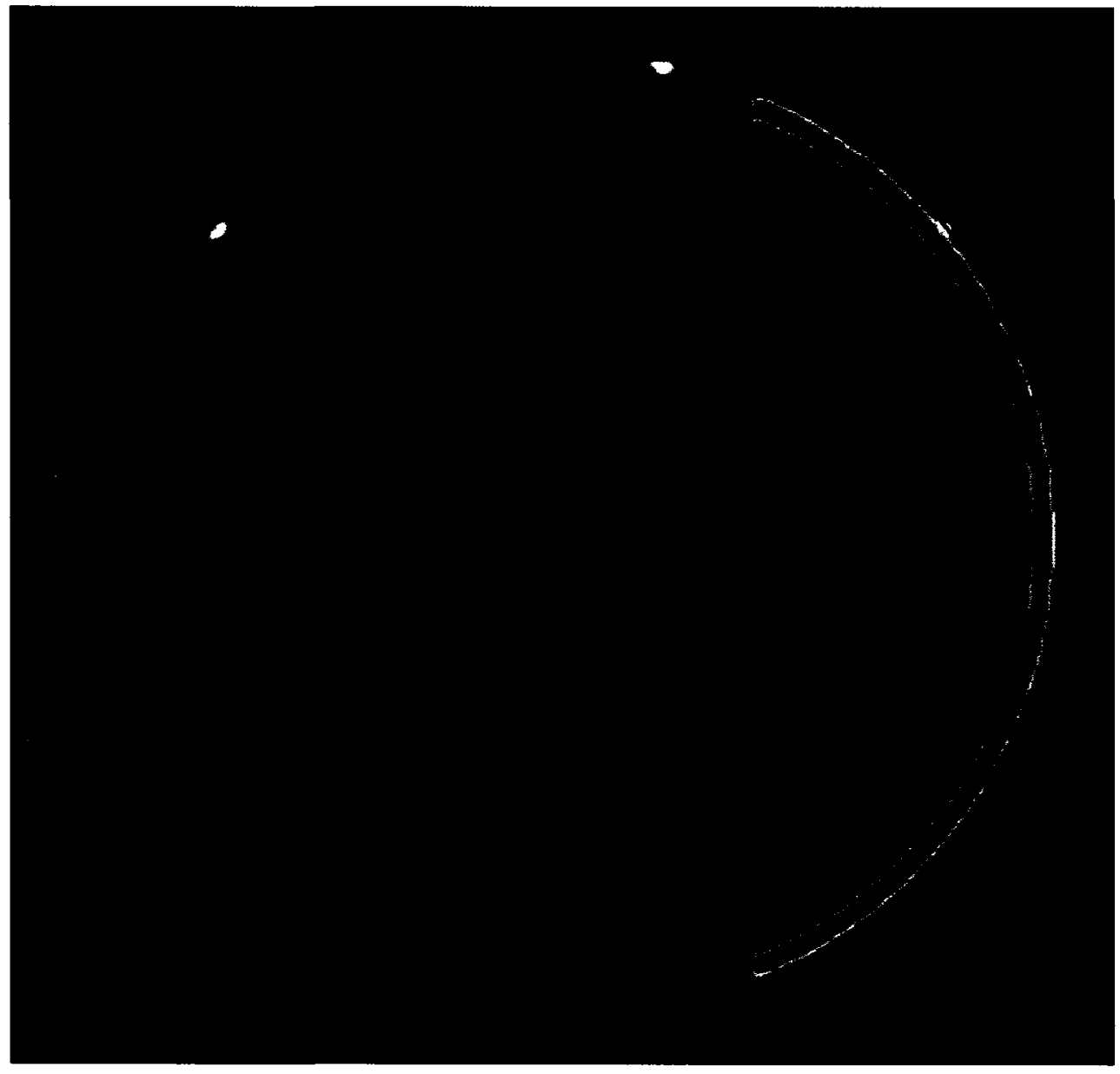

Figure 4.3: Transverse view of phantom showing treatment planning contours with PTVs located at the surface (blue) and $2 \mathrm{~mm}$ (yellow), $4 \mathrm{~mm}$ (cyan), $6 \mathrm{~mm}$ (magenta), $8 \mathrm{~mm}$ (orange), and $10 \mathrm{~mm}$ (purple) inside the surface. Organ at risk (OAR) is shown in green. 
Table 4.1: Optimization parameters entered into the tomotherapy planning software for the planning target volume (PTV) and organ at risk(OAR)

\begin{tabular}{lcc}
\hline \hline Parameter & PTV & OAR \\
\hline Maximum Dose & $50 \mathrm{~Gy}$ & $30 \mathrm{~Gy}$ \\
Maximum Dose Penalty & 10 & 10 \\
DVH Volume & $97 \%$ & $20 \%$ \\
DVH Dose & $50 \mathrm{~Gy}$ & $20 \mathrm{~Gy}$ \\
DVH Dose Penalty & & 10 \\
Minimum Dose & $50 \mathrm{~Gy}$ & \\
Minimum Dose Penalty & 10 & \\
\hline \hline
\end{tabular}

is divided into discrete voxels. Tomotherapy allows the user to choose the resolution of the dose calculation grid. On the 'fine' setting the grid comprises $256 \times 256$ voxels in the $\mathrm{X}-\mathrm{Z}$ plane, so the $\mathrm{X}$ and $\mathrm{Z}$ dimensions of the voxels will depend on the planning $\mathrm{CT}$ field of view. In the Y direction, the number and thickness of the voxels will correspond to the number and thickness of the slices in the planning CT. With a CT slice thickness of $3 \mathrm{~mm}$ and a $50 \mathrm{~cm} \times 50 \mathrm{~cm}$ field of view, and using the 'fine' calculation grid, the voxels used for tomotherapy treatment planning will have dimensions of approximately $1.9 \mathrm{~mm} \times 1.9 \mathrm{~mm}$ $\times 3 \mathrm{~mm}$.

Each voxel is assigned a mass density which is calculated from the CT dataset. The CT 
dataset assigns a value in Hounsfield units (HU) to each voxel:

$$
H U_{x}=\frac{\mu_{X}-\mu_{H_{2} O}}{\mu_{H_{2} O}} \times 1000
$$

where $\mu_{X}$ and $\mu_{\mathrm{H}_{2} \mathrm{O}}$ are the linear attenuation coefficients of material $\mathrm{X}$ and water respectively. Consequently, the lowest possible value on the Hounsfield scale is -1000 for a completely non-attenuating material. In theory, this would mean a perfect vacuum, though for the $120 \mathrm{kV}$ X-rays used for CT scans, $\mu_{\text {air }}$ is also effectively 0.

Though linear attenuation coefficients and density are closely related, the exact relationship depends on both the material being scanned and the energy of the beam. The result is a somewhat non-linear relationship between the image value (CT number, specified in $\mathrm{HU}$ ) and material density. In practice, the conversion is made using a calibration table (plotted in Figure 4.4) consisting of measured image values for several references of known density. Points in between measured values are determined by linear interpolation.

The CT scan data used for our measurements is stored as a 12-bit unsigned integer, with a possible range of $0-4095$. Since the Hounsfield scale extends to -1000 , the integer value is simply shifted by 1024 resulting in a range of possible CT numbers from -1024 to 3071 (even though CT values less than -1000 are not technically valid for ordinary materials, since they correspond to a negative linear attenuation coefficient).

Figure 4.5 shows a histogram of the CT values for 500000 voxels containing only air. The voxels have a range of CT values from -1024 to approximately of -970 . The aircontaining voxels have an average CT value of -998 with a standard deviation of about 8 . 


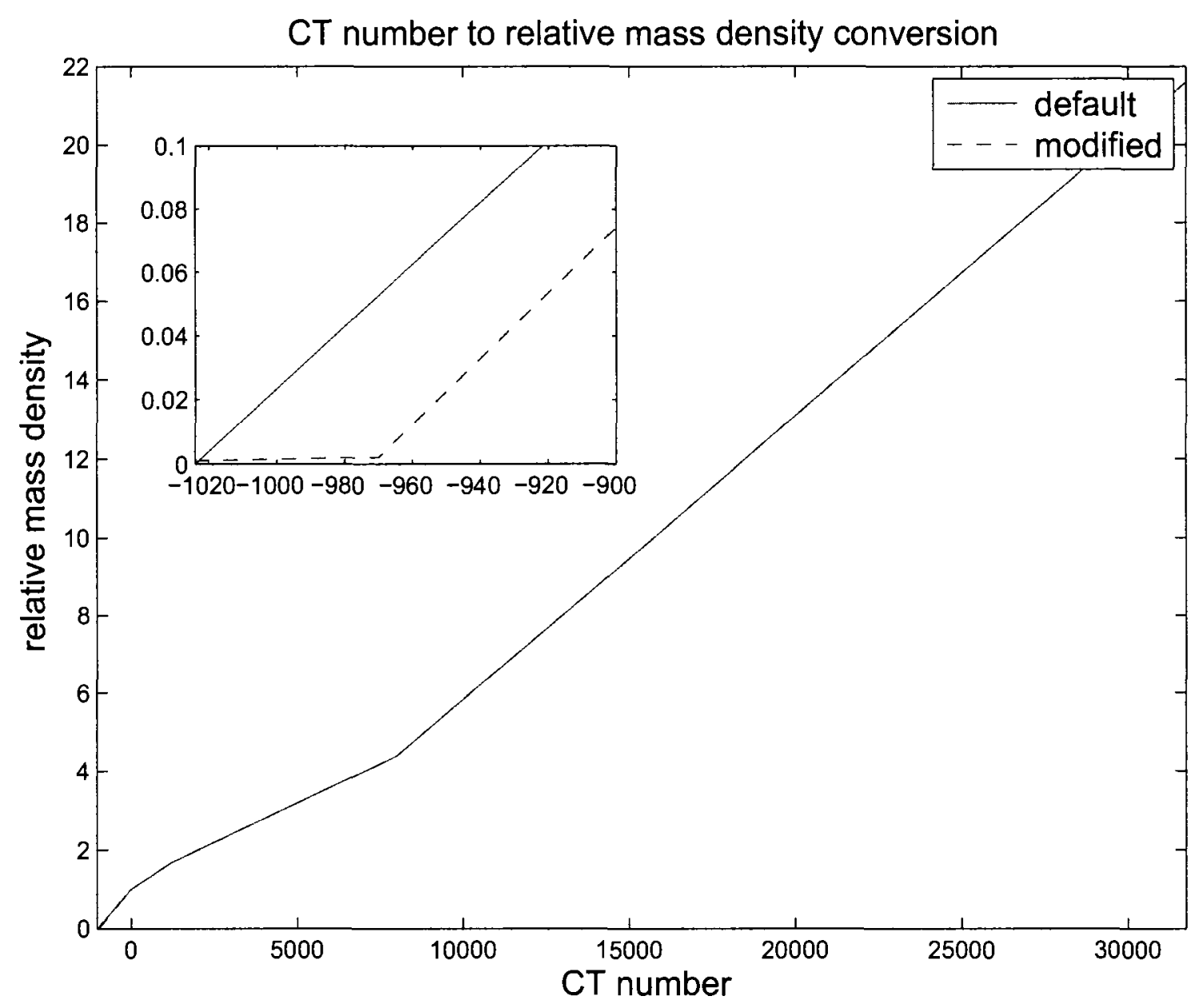

Figure 4.4: Plot of the CT number-to-relative mass density conversion table showing the default and modified table. Inset shows the region where the table has been modified to force the density of the air voxels to more accurately reflect the true density of air. 
The spread of values is due to noise in the CT detector array and electronics.

The original CT data has a resolution of $512 \times 512$ in the X-Z plane, but this is downsampled to a resolution of $256 \times 256$ when the data is imported into the tomotherapy system. Thus, each voxel used in the tomotherapy planning system is in fact an average of four CT voxels. This averaging reduces (by a factor of 2) the spread in CT values due purely to random noise.

The current table being used for tomotherapy planning assigns relative mass densities to these voxels based on linear interpolation between two points on in the calibration table: a relative density of 0 for a $\mathrm{CT}$ value of -1024 , and 1.000 at 0 . A quick calculation will show that the 'air voxels' with CT values ranging from -1024 to about -970 will be assigned relative densities ranging from $0.000-0.053$, with a mean value of about 0.023 . The accepted value for the relative density of air is 0.001205 , implying that the planning system is assigning voxels containing air a relative density that is too high by a factor of approximately 20. For the calculation of dose inside the patient where the relative mass density is on average a thousand greater than that of air, this is likely to be of negligible significance. However, the calculation of dose directly adjacent to the air may be less tolerant of significant errors in the assignment of relative mass density to adjacent voxels. Since the primary focus of this work involved delivering and measuring dose at shallow depths, the impact of accurately specifying the relative density of the voxels containing air was assessed before generating the treatment plans used to measure the behavior of the tomotherapy system for shallow-depth PTVs. 


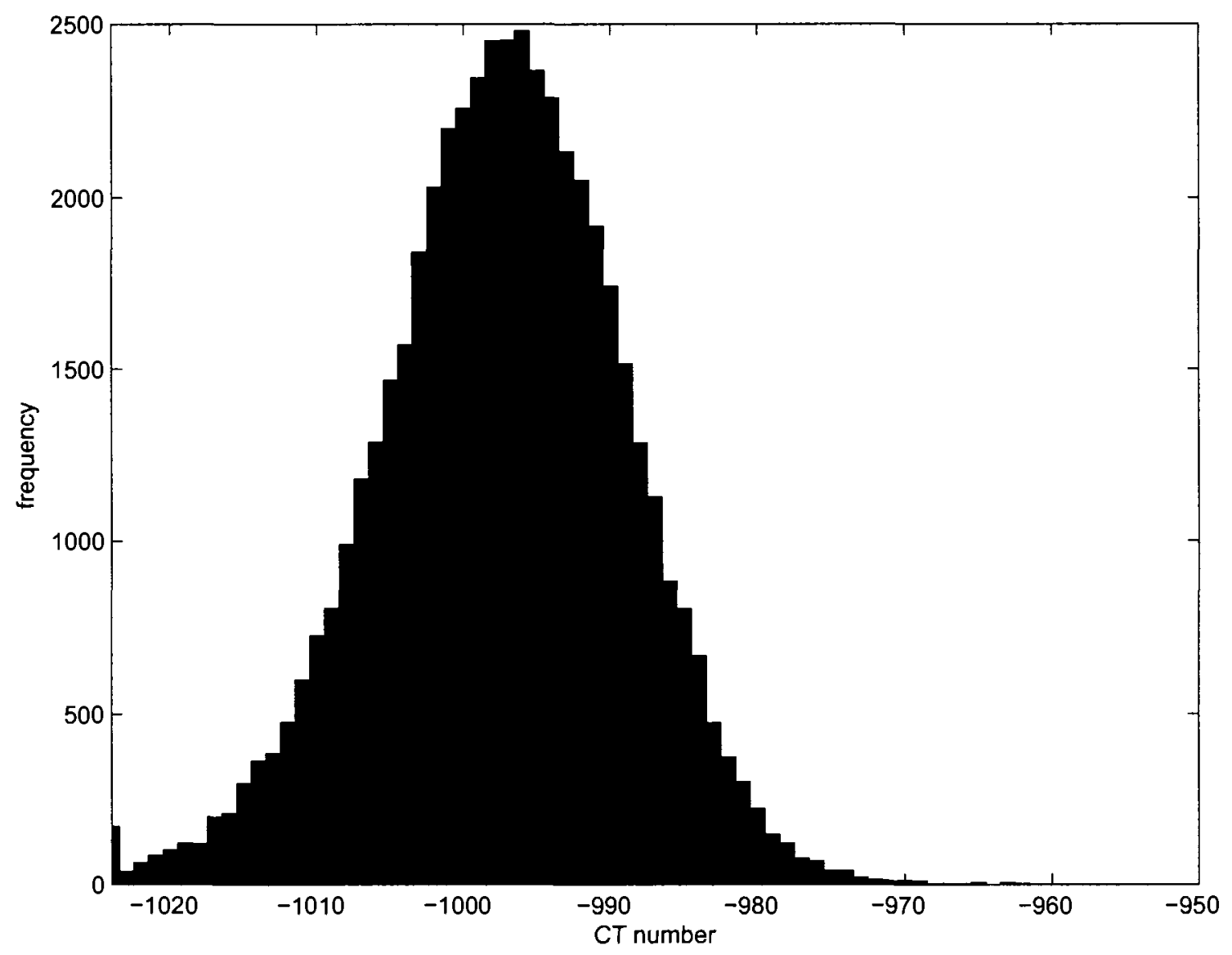

Figure 4.5: Histogram of the CT values for 500000 voxels containing air. 


\subsubsection{Measurements}

For all measurements, the film was inserted between the two halves of the phantom (Figure 4.6, resulting in a record of the dose in the X-Y plane passing through the thickest portion of the target volume.

Following exposure and development, the optical density was measured and digitized by a VXR-16 DosimetryPRO scanner (Vidar, Herndon, VA).

To measure the sensitivity of the tomotherapy planning system to the relative density assigned to the air, we created two plans, one calculated using the default image valueto-density table and the other calculated using a modified table. The modified conversion table includes two extra points at -1023 and -970 to force CT values ranging from -1023-970 into the relative density range of $0.001-0.002$ (Figure 4.4 ). Both plans used identical planning contours with the PTV extended all the way to the surface of the phantom, where the plan would be most sensitive to changes affecting the density of the air surrounding the phantom.

As will be shown below, the measured dose showed better agreement with the plans created using the modified CT-to-relative density table. Therefore, the modified version of the table was used to calculate the treatment plans for the remaining experiments. To evaluate the consequences of changing the depth of the PTV, plans were created for each of the PTVs described above in Section 4.2.3.

To measure the effects of misaligning the patient, dose was delivered for two different 


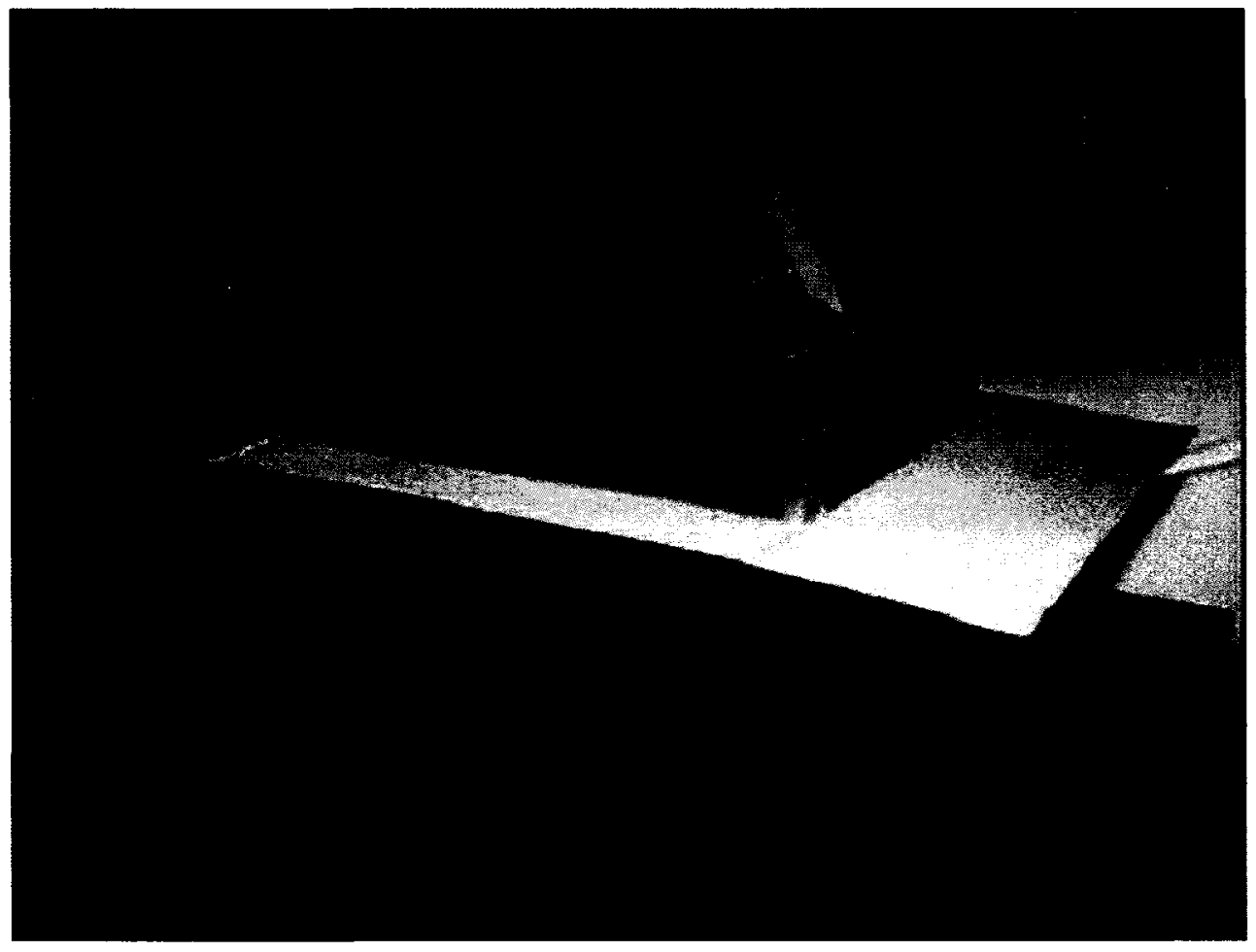

Figure 4.6: Cylindrical phantom with film in $\mathrm{X}-\mathrm{Y}$ plane. As depicted, the positive $\mathrm{Z}$ axis is in the positive vertical direction, the positive $\mathrm{X}$ axis is to the right, and the positive $\mathrm{Y}$ axis is away from the viewer (into the bore of the gantry). 
plans with the phantom misaligned along the $\mathrm{X}$ axis. Each of the plans was delivered with the phantom horizontally shifted by $4 \mathrm{~mm}$ in the negative $\mathrm{X}$ direction, bringing the target closer to the central axis of the linear accelerator, and with the phantom shifted $4 \mathrm{~mm}$ in the positive $\mathrm{X}$ direction, moving the target away from the central axis. This was carried out for the plan with a $0 \mathrm{~mm}$ surface to PTV margin, assumed to be the worst-case scenario with respect to the difficulty of achieving the desired dose and sensitivity to even small misalignments. This experiment was also done for the plan created with a $4 \mathrm{~mm}$ surface to PTV margin.

The plans were delivered to the phantom, using a sheet of EDR2 film in the X-Y plane to measure the dose. For each plan, a small piece of GafChromic film was also taped to the external surface of the phantom just above the EDR2 film plane to measure the surface dose.

For each plan, the film measurements were repeated five times, with the phantom realigned each time, in an attempt to reduce the likelihood that any differences observed between the two plans were due to random variation between tests.

Each day that plans were delivered, the calibration curve was generated using the calibration procedure described above. However, even with daily film calibration, there was some discrepancy of up to $5 \%$ between the dose measured by the film and the dose predicted by the software, even for the region of uniform dose a few centimeters below the surface of the phantom. Therefore, the measured and calculated doses were normalized to the uniform region within the phantom where the software has been verified to accurately 
calculate dose. This was judged to be acceptable in this study, where the intent was to determine the ability of the tomotherapy system to accurately calculate and deliver dose to shallow-depth PTVs, rather than to investigate the absolute dose accuracy of the system.

Alignment of the film to the calculated dose matrix was accomplished using pin pricks in the film to mark positions of the lasers relative to the film. However, close comparison of the resulting alignment between the plan and the measured dose suggested that the alignment accuracy using pin pricks alone was somewhat limited. The pin pricks were made at the location of pen marks on the film marking the position of the alignment lasers in the treatment room which are calibrated to line up to the isocenter of the gantry. The laser beam itself has a finite width, making it difficult to mark its location on the film to a precision of better than about $0.5 \mathrm{~mm}$. The film itself may move slightly within its envelope from the time the exposure is made and the laser positions are marked, and the time when the pin pricks are made. Together, these limit the precision of the film alignment resulting in an uncertainty of about $\pm 1 \mathrm{~mm}$.

Two observations suggest that the alignment of the film may be improved somewhat by directly aligning the measured profile with the plan. First, the so-called 'thread effect' is visible on both the plan and the film, allowing precise alignment in the y-direction. (The 'thread effect' refers to the ripple pattern in the dose measured along the y-axis due to the junctioning between adjacent beams as the gantry rotates around the patient. ${ }^{42}$ ) Second, at the surface of the phantom there is a visible discontinuity in the dose falloff which permits alignment along the $\mathrm{x}$-axis. Careful matching of the thread artifact and the surface 
discontinuity made it possible to align the film to an accuracy of better than $1 \mathrm{~mm}$.

\subsection{Results and Analysis}

\subsubsection{Density Conversion Table}

The dose predicted by the tomotherapy planning software and the measured doses using the unmodified density table are plotted in Figure 4.7(a). Figure 4.7(b) shows the same data using the modified density table. The predicted and measured dose for both figures shows a similar shape. The plan asks for a uniform dose of $200 \mathrm{cGy}$ (per fraction) extending all the way to the surface of the skin. In both cases, the volume within $3 \mathrm{~mm}$ of the surface of the phantom is slightly under dosed. Slightly deeper, at a depth of about $5-7 \mathrm{~mm}$, is a region that is severely overdosed, and following that is a region of relatively uniform dose matching the prescription dose.

The calculated and delivered dose deep inside the patient is insensitive to the changes made to the relative density table. This is expected, since the changes to the density table only affected the voxels containing air. Inside the phantom at a depth of more than 2 $\mathrm{cm}$, virtually none of the dose comes either indirectly from photon interactions in the air surrounding the phantom or from electrons that could have been scattered into or out of the air. The dose distribution there is thus insensitive to the changes to the density table, provided that the changes do not in any meaningful way impact the calculated photon fluence inside the phantom. Since the density of air (used for calculation) is in either case, 


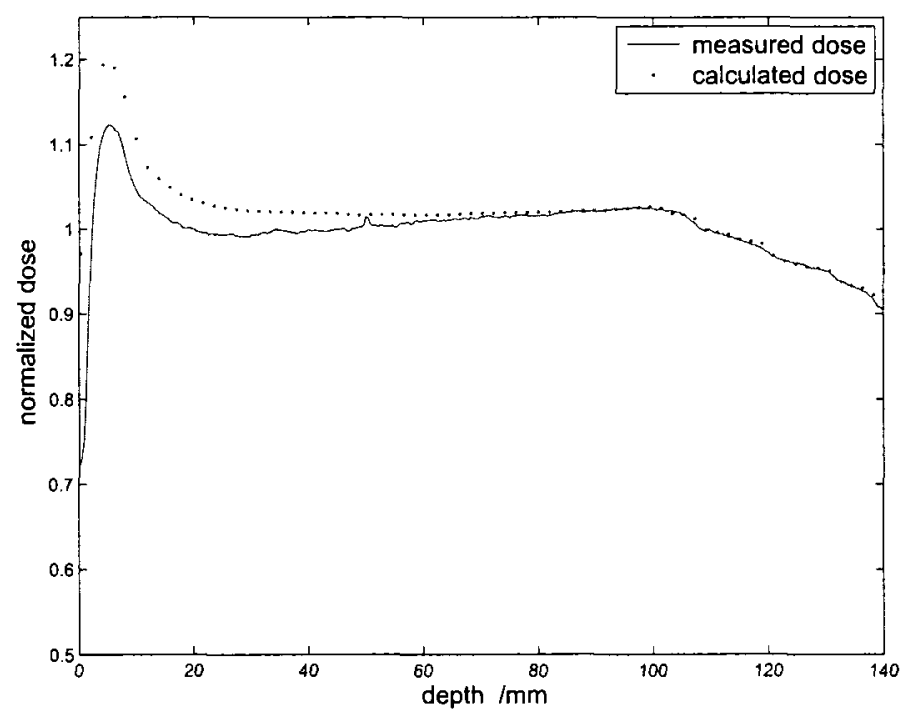

(a) Unmodified relative density table

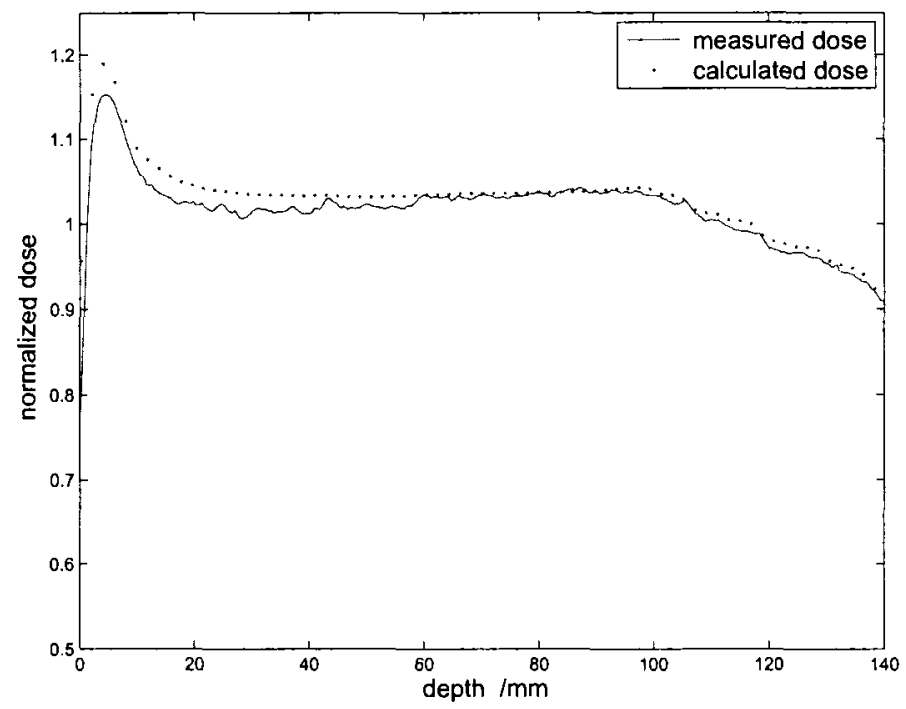

(b) Modified relative density table

Figure 4.7: Calculated and measured dose profiles for unmodified and modified relative density tables. Dose has been normalized to the prescription dose of 200cGy. 
at most, only about $2 \%$ of the density of water or tissue, this condition holds.

The peak dose is overestimated in both cases, though the discrepancy is much larger when using the unmodified table. In this case, the calculated peak is $19.4 \%$ about the prescription dose and the measured peak is $11.8 \%$ above $200 \mathrm{cGy}$, a discrepancy of more than $7 \%$. The difference between the calculated and measured dose is only half as large when using the modified table, where the peak was again calculated to be about $19.0 \%$ too high, but the measured dose was $15.3 \%$ higher than the prescribed dose. Both plots also show the delivered dose to be less than calculated 'behind' the peak, with the results from the plan created with the unmodified table again showing greater discrepancy between predicted and measured dose.

Several factors could be contributing to the differences that we observe. The first is a consequence of using scaled pre-calculated dose convolution kernels to predict dose. These kernels are calculated using Monte Carlo simulation methods and give a relatively accurate description of dose deposition inside a homogeneous medium. These kernels are then scaled by density to account for inhomogeneities in the mass density of the target. Though generally accurate, this scaling cannot accurately calculate dose in regions of lateral electronic disequilibrium. The reasons for this are discussed in the introduction to this chapter. The result is an overestimation of dose in a region of dense tissue near a boundary with air or significantly less dense tissue. This could account for the software predicting a dose higher than that actually measured for both the modified and unmodified mass density tables. 
Artificially setting the mass density of the air too high, as is the case when using the unmodified table, exacerbates the overestimation of dose near the surface of the phantom. Even though a relatively small amount of energy is released by photon interactions in the air around the phantom, setting the air density too high by a factor of 20 would result in the calculated TERMA for the volume of air surrounding the phantom being too high by the same factor. Since the density of the air is still very low relative to the density of the phantom, the scaled dose spread kernels would nearly all intersect with the phantom, depositing some finite amount of energy near the phantom surface. This effect could account for the larger overestimate of dose seen in the case of the unmodified relative electron density table.

\subsubsection{Effect of PTV depth on dose profile}

Figure 4.8 shows the planned and measured doses in the X-Y plane for the case where the PTV depth is $0 \mathrm{~mm}$. The rind of overdosing near the surface of the phantom can be clearly seen on both the plan and the film.

Profiles of the dose distributions in Figure 4.8 are shown in figure 4.9. From figure 4.9(a) it is clear that the presence of the overdosed region is accurately predicted by the tomotherapy software. The exact height of the dose peak is slightly overestimated -238 cGy (19\% above prescribed dose) compared with a measured value of 233 cGy $(17 \%)$. This corresponds to an overestimation of approximately $2 \%$. This is consistent with the tendency of the convolution/superposition algorithm to overestimate dose in dense material near an 


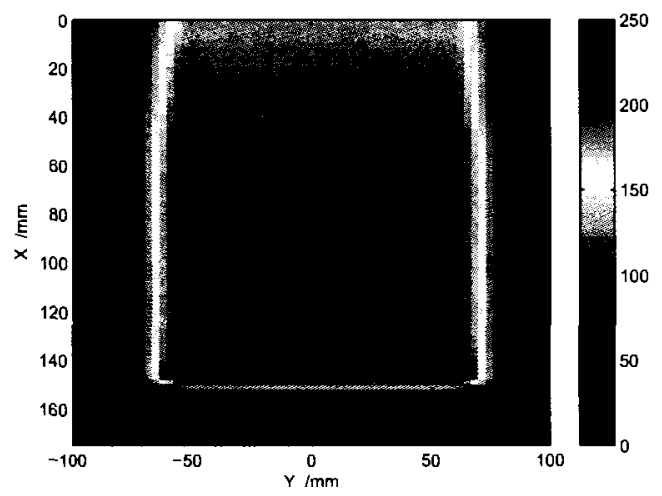

(a) Calculated

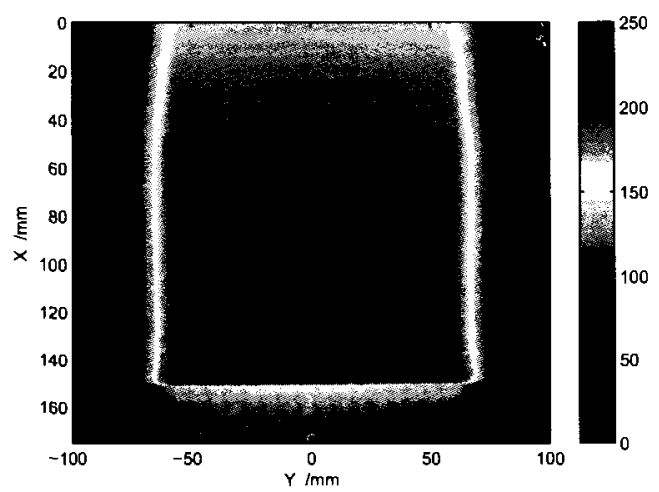

(b) Measured Dose

Figure 4.8: Calculated and measured dose distributions in the $Z=0$ plane with a PTV depth of $0 \mathrm{~mm}$. Color scale in cGy. $\mathrm{X}$ and $\mathrm{Y}$ are in tomotherapy coordinates, measured from the isocentre of the treatment plan. Note that the thread effect is slightly visible in the calculated dose and clearly discernable in the measured dose distribution 
interface with air (or any substance of significantly lower density). Placing the PTV at the surface of the phantom seems to be resulting in a substantial volume of the phantom receiving a dose more than $15 \%$ greater than the prescribed dose.

Figure 4.9 (b) shows the calculated and measured dose along the $Y$ axis at the peak of the dose distribution about $5 \mathrm{~mm}$ inside the surface of the phantom. Evident from this figure are the limitations of the tomotherapy software when it comes to predicting the thread effect. This is primarily a result of averaging the dose over the entire volume of each voxel in the dose calculation grid. A jaw width of $25 \mathrm{~mm}$ and a pitch of 0.3 gives a separation between each 'thread' of $7.5 \mathrm{~mm}$. Since the calculation grid has a resolution of only $3 \mathrm{~mm}$ along the $\mathrm{Y}$ axis, small artifacts in the dose distribution, like the thread effect, will be partially smoothed out. At the peak, the ripple in the dose distribution due to the thread effect has an amplitude of about $8 \mathrm{cGy}$, or approximately $3.5 \%$ of full dose.

Dose profiles for PTV margins of $2 \mathrm{~mm}, 4 \mathrm{~mm}, 6 \mathrm{~mm}$, and $10 \mathrm{~mm}$ are plotted in Figures 4.10-4.13. Even separating the PTV from the surface by only $2 \mathrm{~mm}$-approximately the thickness of a single voxel-is sufficient to substantially reduce the excess dose to the patient. With the PTV $2 \mathrm{~mm}$ inside the surface of the phantom, the predicted dose peak drops to about $106 \%$, and the measured dose peak to only $103 \%$. With the PTV at $4 \mathrm{~mm}$ or $6 \mathrm{~mm}$, there is no evidence of significant dose dumping.

The thread effect becomes more significant at at distances farther from the central axis of the bore, and may be of some concern when treating breast cancer. Figure 4.14 shows the difference in the magnitude of the thread effect between a point approximately $100 \mathrm{~mm}$ 


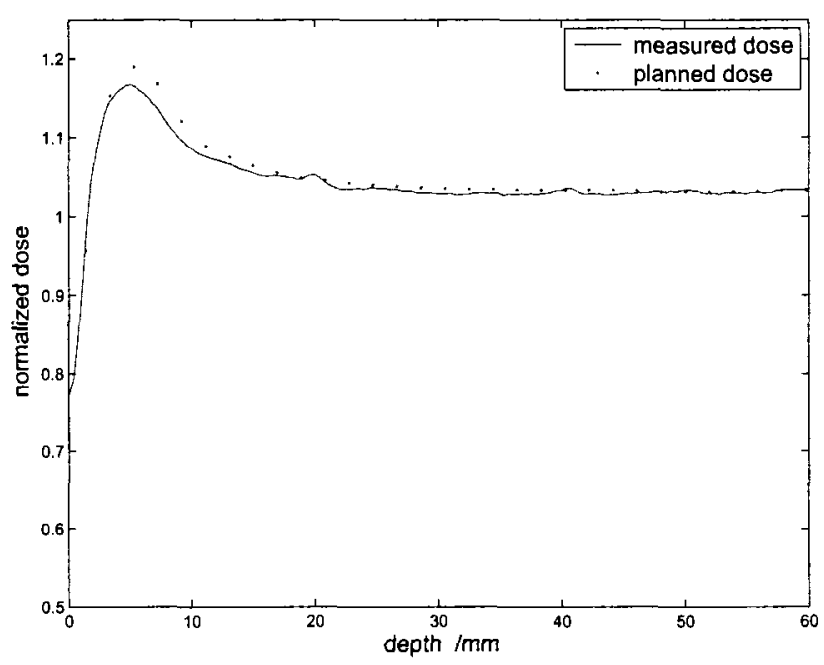

(a) Dose profile along $\mathrm{X}$ axis at centre of phantom

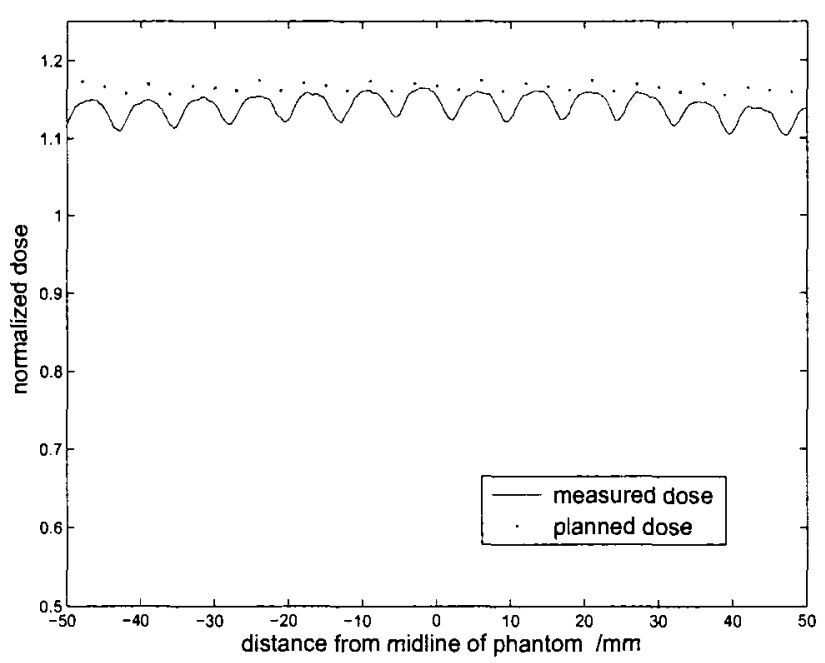

(b) Dose profile along $\mathrm{Y}$ axis at peak $(5 \mathrm{~mm}$ inside sur-

face)

Figure 4.9: Dose profiles in the $\mathrm{X}$ and $\mathrm{Y}$ directions for a PTV depth of $0 \mathrm{~mm}$. Dose has been normalized to the prescription dose of 200cGy. 


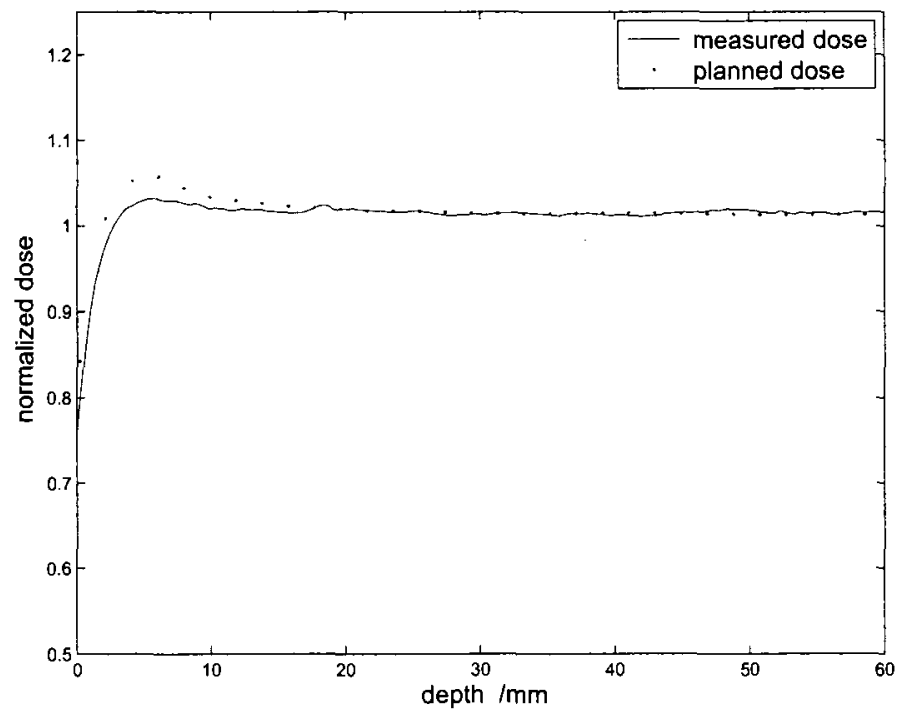

Figure 4.10: Dose profile along $X$ axis at centre of phantom for PTV depth of $2 \mathrm{~mm}$.

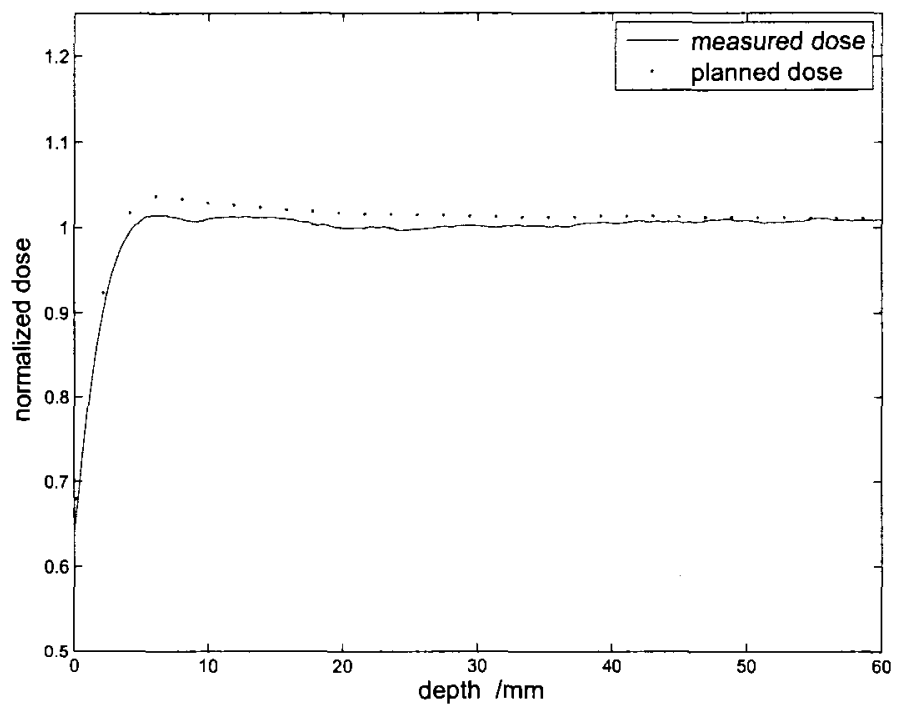

Figure 4.11: Dose profile along $X$ axis at centre of phantom for PTV depth of $4 \mathrm{~mm}$. 


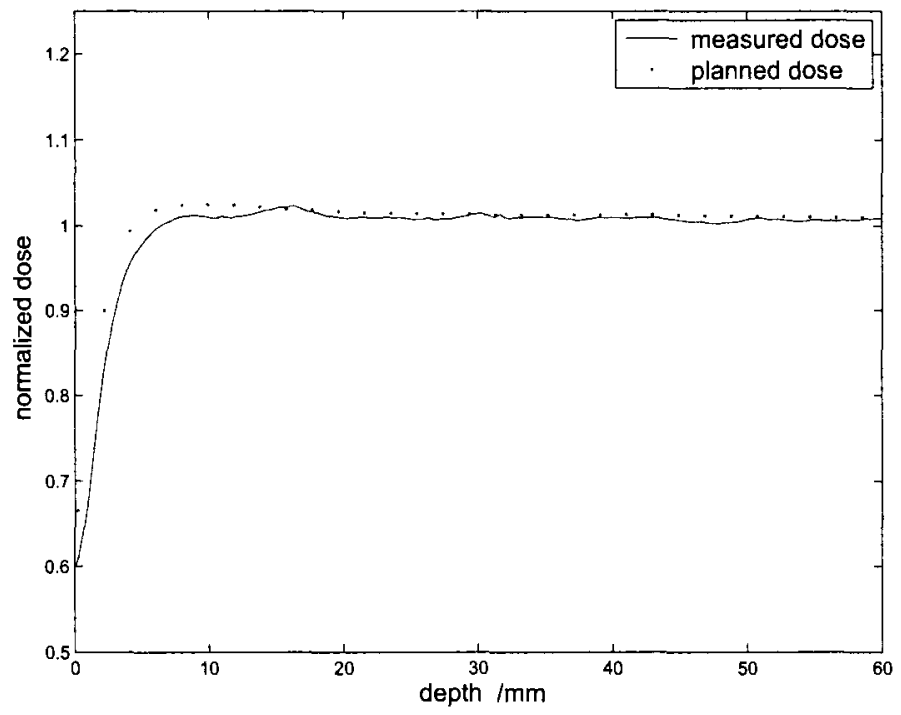

Figure 4.12: Dose profile along $\mathrm{X}$ axis at centre of phantom for PTV depth of $6 \mathrm{~mm}$

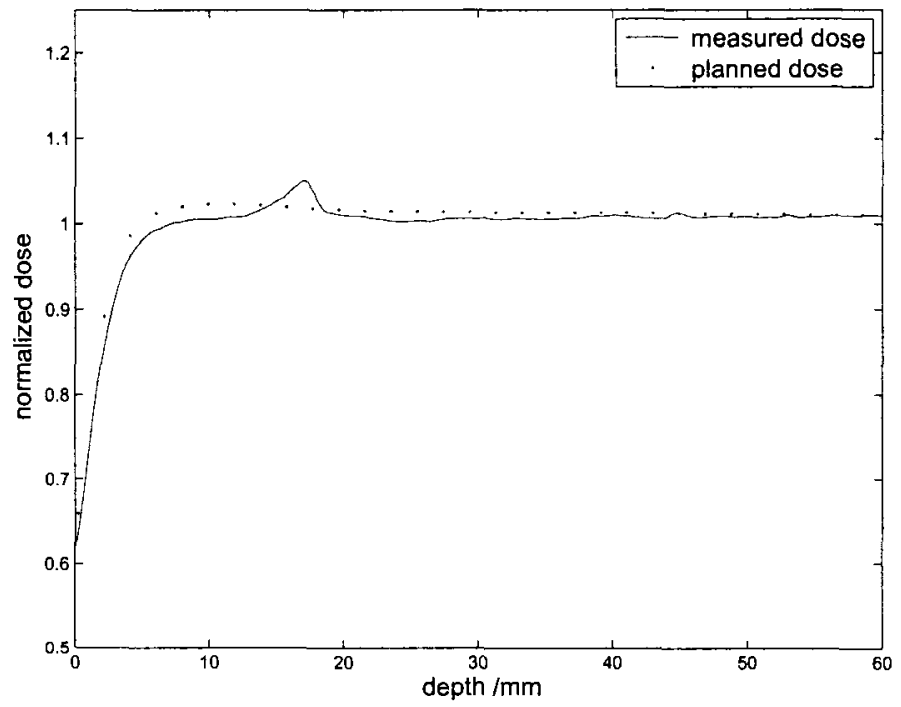

Figure 4.13: Dose profiles along $X$ axis at centre of phantom for PTV depth of $10 \mathrm{~mm}$. 
from the axis and another approximately $145 \mathrm{~mm}$ from the axis. At $145 \mathrm{~mm}$ from the axis the ripple has an amplitude of about $3 \%, 45 \mathrm{~mm}$ closer to the axis, the ripple is less than 1\%. In many cases, the borders of an actual breast will be even farther from the axis, more than $20 \mathrm{~cm}$ in the case of some women.

In every case, the exact values of the reported results are specific to a particular set up and choice of optimization parameters. Changing either of these, particularly the latter, will result in a different treatment plan and a different degree of over- or underdosing of the patient. However, the results will remain qualitatively the same. Placing the PTV at the surface results in an undesirable dose distribution at shallow depths.

\subsubsection{Surface Dose Measurements}

Table 4.2 shows the predicted dose for the voxels just inside the surface of the phantom, the predicted dose for the voxels just outside the surface of the phantom, and the surface dose measured using Gafchromic film. Measurements were made for each of the PTV-to-surface distances between $0 \mathrm{~mm}$ and $10 \mathrm{~mm}$.

From this data it can be seen that the surface dose as measured using Gafchromic film was consistently approximately midway between the dose to the voxels lying just outside and just inside the surface of the phantom, though the large discrepancy in dose between the two makes this a relatively loose constraint. In all cases, the measured dose fell at or just below the average of the dose calculated for the two voxels adjacent to the surface of the phantom. The data also shows that with the PTV located at the surface, the surface dose 


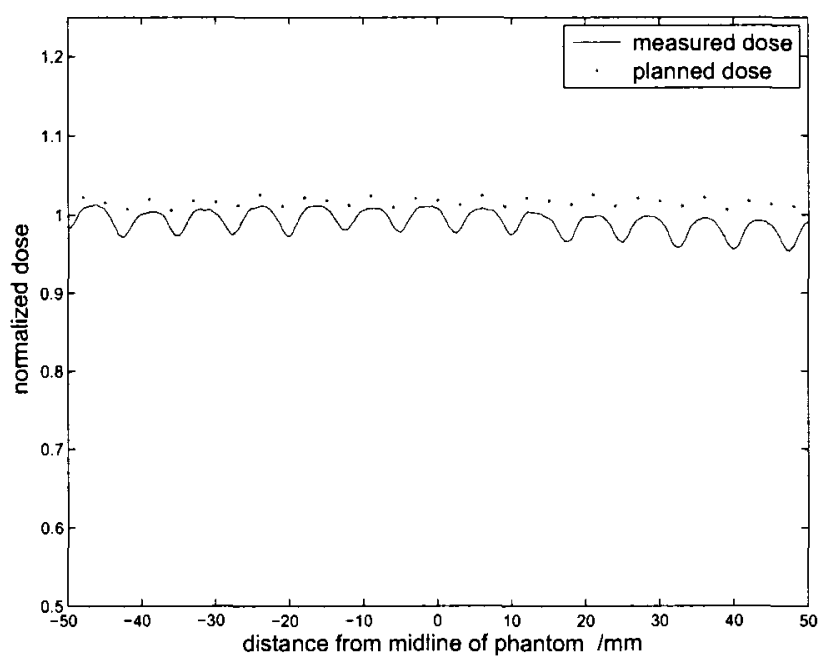

(a) $5 \mathrm{~mm}$ depth

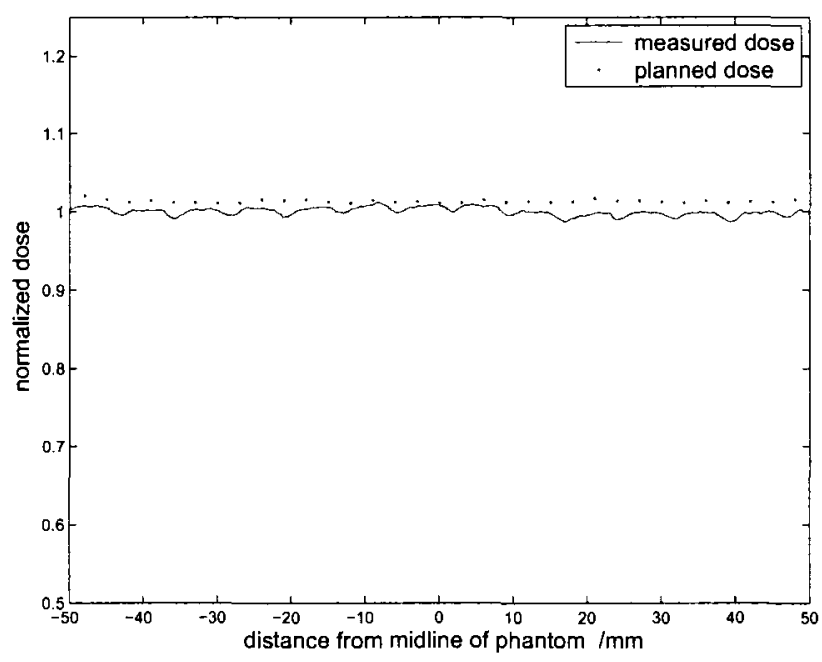

(b) $50 \mathrm{~mm}$ depth

Figure 4.14: Dose profile parallel to axis of gantry at depths of $5 \mathrm{~mm}$ (145 $\mathrm{mm}$ from axis) and $50 \mathrm{~mm}$ (100 $\mathrm{mm}$ from axis). The thread effect becomes much more pronounced farther away from the axis. 
Table 4.2: Surface dose predictions for voxels just inside and just outside the phantom surface and Gafchromic measurements, in cGy

\begin{tabular}{lccc}
\hline \hline \multirow{2}{*}{$\begin{array}{l}\text { PTV Depth } \\
(\mathrm{mm})\end{array}$} & \multicolumn{2}{c}{ Calculated } & Measured \\
\hline 0 & 192 & 132 & 152 \\
2 & 168 & 112 & 141 \\
4 & 136 & 88 & 99 \\
6 & 132 & 88 & 100 \\
8 & 132 & 84 & 99 \\
10 & 132 & 84 & 103 \\
\hline
\end{tabular}

is about $75 \%$ of the prescribed dose. Retracting the PTV by $2 \mathrm{~mm}$ reduces this slightly to about $70 \%$ of the prescribed dose. With the PTV at a depth of $4,6,8$, or $10 \mathrm{~mm}$, the measured surface dose was relatively constant at about $50 \%$ of the prescribed dose.

\subsubsection{Sensitivity of dose profile to misalignment of phantom}

Figure 4.15 shows the results of delivering treatment plans with the phantom misaligned.

For the plan where the border of the PTV was located at the surface of the phantom, the software predicted a dose peak of about $238 \mathrm{cGy}$ (19\% above prescription). Shifting the phantom laterally, (i.e. moving the phantom to the right, or in the positive $\mathrm{X}$ direction), results in a dose peak even higher than calculated - about 248 cGy (24\% too high) - 
and seemingly slightly wider and deeper than predicted. The consequences of misaligning the phantom in this way are even more serious when compared to the dose actually delivered with the phantom aligned correctly (Figure 4.9(a)), where the peak dose was only 233 cGy (17\%). Misaligning the phantom medially (i.e. to the left, or in the negative $\mathrm{X}$ direction), nearly eliminates the dose peak altogether and results in a dose profile similar to that obtained for surface to PTV margins of about $2 \mathrm{~mm}$ as in Figure 4.10. In this case the high fluence portion of the beam intended to deliver almost full dose to the surface of the phantom misses the phantom entirely. The effects of the high photon fluence in the air near the surface of the phantom is visible on the film, though the exact dose delivered to the air in that region can not be reliably determined from the film.

The consequences of misalignment when a surface to PTV margin of $4 \mathrm{~mm}$ is used are less dramatic. As Figure 4.15(b) shows, shifting the phantom in a lateral direction resulted in a slightly lower dose near the surface of the phantom and a small dose peak of about the same magnitude as the software predicted, but perhaps $2 \mathrm{~mm}$ deeper. The first $5 \mathrm{~mm}$ of tissue also receive about $15 \%$ less dose then with the phantom correctly aligned. A medial shift of $4 \mathrm{~mm}$ resulted in almost no difference in the dose distribution near the surface of the phantom, except for a slightly lower (4\%) dose at a depth of $5 \mathrm{~mm}$.

Although not shown, for both plans, the misalignment of the phantom resulted in a nearly identical misalignment of the dose profile at the 'back' side of the PTV, as would be expected. 


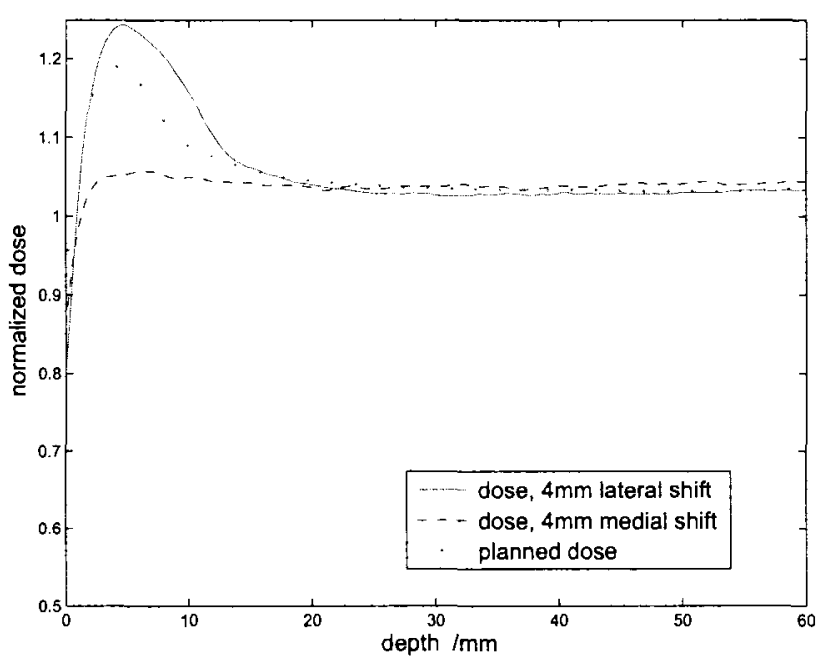

(a) PTV depth of $0 \mathrm{~mm}$

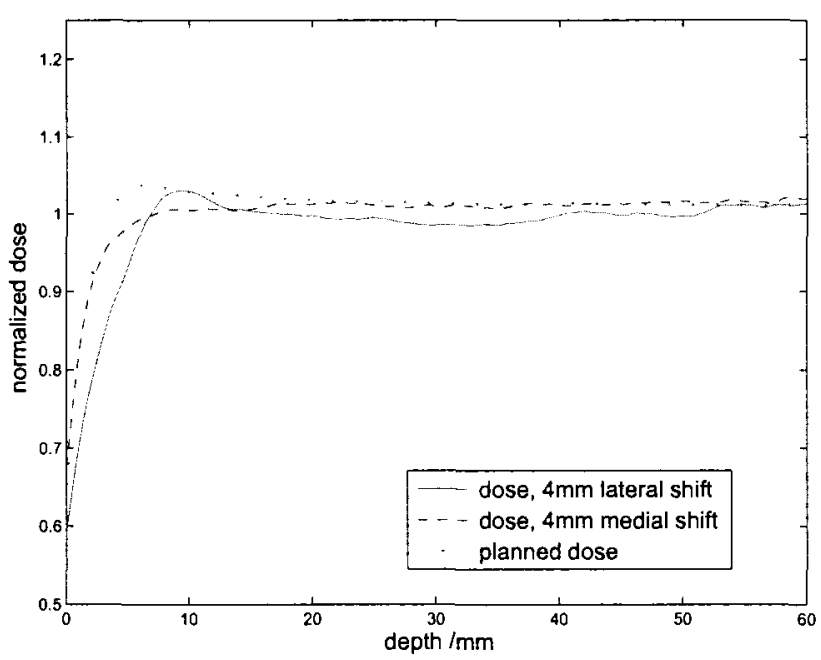

(b) PTV depth of $4 \mathrm{~mm}$

Figure 4.15: Dose profiles showing the consequences of misaligning the phantom by $4 \mathrm{~mm}$ laterally or medially long the X axis. Results are shown for the plans with the PTV at the surface and with the PTV at a depth of $4 \mathrm{~mm}$. 


\subsection{Conclusions}

\subsubsection{CT number to mass density curve}

Modifying the relative electron density table so that the CT-to-mass density conversion more accurately reflects the true mass density of the voxels containing air was shown to result in more accurate prediction of the dose near the surface of the phantom. Therefore, all other work was done using the modified density curve. Tomotherapy is one of the few treatment planning systems that directly takes into account photon interactions in the air surrounding the phantom. This being the case, accurate dose calculation near the surface of the phantom requires that the air be accurately modeled. We've shown that mapping CT number to density using only a single point (CT:-1024 to a relative density of 0 ) results in an overestimate of the air density, and that this in turn leads to an overestimate of the dose at superficial depths.

\subsubsection{PTV Depth and Dose Dumping}

As expected, and predicted by the planning software, placing the PTV too close to the surface does indeed result in dose dumping — a 'rind' of overdosed tissue just below the surface of the phantom. The tomotherapy software slightly overestimates the intensity of the dose dumping. In fact, near the edge of the phantom, or $150 \mathrm{~mm}$ from the central axis of the tomotherapy unit, the miscalculation of the dose at a specific point due to the interaction of the thread effect and voxel averaging was at least as great (3.5\%) as the overestimation 
of dose near the surface (2-3\%). Based on our results, the PTV should never be placed at the surface of the breast for whole breast irradiation. Using a margin of only $2 \mathrm{~mm}$ between the PTV and the surface was enough to reduce the magnitude of the dose dumping to about $3 \%$, and PTV depths of $4 \mathrm{~mm}$ or more eliminated all traces of the effect.

None of the plans was capable of delivering dose without underdoseing the first 2-3 $\mathrm{mm}$ of skin. For those patients where the disease has spread to the skin itself and full dose to the skin is indicated, bolus will be necessary.

Measurements using Gafchromic film confirm that surface dose may be estimated from the calculated dose to the voxels in the vicinity of the surface. However, partial volume effects virtually guarantee that any such predictions are subject to large uncertainties. The actual surface dose was very sensitive to the depth of the PTV when the PTV was within $4 \mathrm{~mm}$ of the surface. At a depth of $4 \mathrm{~mm}$ or more, the surface dose was unchanged by changes to the PTV depth.

\subsubsection{Sensitivity to Misalignment}

When the PTV is at the surface of the phantom, any misalignment may result in a dose distribution substantially different from that which is expected. Specifically, this means a dose peak that is slightly higher than predicted if the phantom is misaligned in one direction or a reduction of the dose peak if the phantom is moved the other way. In practice this is probably of limited significance since there are other reasons, as discussed above, for not extending the PTV all the way to the surface. With the PTV at a depth of $4 \mathrm{~mm}$, the impact 
of misalignment on the superficial dose distribution is measurable, but modest. 


\section{Chapter 5}

\section{Conclusions}

The intent of this research was to evaluate the factors that affect superficial dose distribution when treating breast cancer using tomotherapy. The primary intent was to determine if expanding the treatment beams beyond the surface of the breast (flash) is either necessary or desirable when using tomotherapy to treat breast cancer. Use of flash is standard with traditional methods of breast cancer radiation therapy, but presents difficulties in an inverse planned system like tomotherapy where the planner cannot directly control the extent of the treatment beam.

\subsection{Immobilization}

The first part of the research was intended to determine whether flash was even necessary. During conventional breast radiation therapy the breast is normally immobilized using 
gravity alone. Flash is necessary to deal with residual motion from respiration and small changes in patient position and alignment from one treatment to the next. Tomotherapy requires much more reproducible patient alignment and therefore the patients were placed on a breastboard and immobilized using a thermoplastic shell. Given that this should immobilize the patient more than gravity alone, is flash still necessary to allow for respiratory motion?

Analysis of 4D CT scans for a group of eight patients at The Ottawa Hospital Cancer Centre showed that the total intrafraction displacement of the breast surface was less than $4 \mathrm{~mm}$ for all eight patients and less than $2 \mathrm{~mm}$ for most of the patients. Pre-treatment shift data for the same patients was analyzed to determine the interfraction misalignment (setup error) for comparison. This analysis showed significant random and systematic setup errors of $8.5 \mathrm{~mm}$ and $11.0 \mathrm{~mm}$ respectively. However, since this data reflects only amount of shift applied to the patient before treatment, it cannot be taken to be indicative of the setup error during treatment. Other research which investigated residual setup error using tomotherapy to treat locations other than the breast, and research that measured residual setup error for breast patients using cone-beam CT has reported setup errors in the range of 1-2 mm following patient registration. Future research to confirm that similar results can be achieved for breast patients using the method of immobilization investigated here would be desirable.

Based on this analysis, it is probable that breast tomotherapy will be able to forgo the use of flash and still achieve acceptable dose coverage. 


\subsection{PTV Depth}

The second portion of our research was intended to establish the relationship between the depth of the PTV and the dose distribution near the surface of the patient.

This work demonstrated the deposition of substantial overdosing of $15-20 \%$ just inside the surface when the PTV is extended to the surface of the patient. Maintaining a margin of $4 \mathrm{~mm}$ or more between the PTV and the surface eliminates the overdosing and reduces the surface dose to about $50 \%$ of full dose. Little change is seen in either the skin dose or the dose distribution for depths between 4-8 $\mathrm{mm}$.

As well as eliminating the hot spots seen when the PTV is drawn at the surface, the resulting plan is also less sensitive to small changes in the position of the skin surface when the PTV is drawn $4 \mathrm{~mm}$ or more inside the surface.

\subsection{Recommendations}

The inverse treatment planning system used for tomotherapy does not naturally lend itself to the inclusion of flash when treating breast cancer. However, by immobilizing the patient using a thermoplastic shell and using image guidance to position the patient, the uncertainty in the position of the patient can probably be reduced to the point where flash becomes unnecessary. In fact, treatment planning should be done with the PTV drawn inside the surface of the patient to prevent the deposition of excessive amounts of dose just below the skin of the patient. Margins of approximately $4 \mathrm{~mm}$ between the skin surface and the PTV 
are recommended when planning tomotherapy for breast cancer. 


\section{Bibliography}

[1] Canadian Cancer Society/National Cancer Institute of Canada. Canadian Cancer Statistics 2007, Toronto, Canada, 2007.

[2] Early Breast Cancer Trialists' Collaborative Group. "Effects of radiotherapy and surgery in early breast cancer. An overview of the randomized trials." N. Engl. J. Med. 330 (1995):1444-1455.

[3] A.J. Schouten van der Velden, R. van Vugt, J.A.A.M. van Dijck, J.W.H. Leer, and T. Wobbes, "Local recurrences after different treatment strategies for ductal carcinoma in situ of the breast: a population-based study in the east Netherlands." Int. J. Radiation Oncology Biol. Phys. 69 (2007): 703-710.

[4] M. Overgaard, P.S. Hansen, J. Overgaard, et al., "Postoperative radiotherapy in highrisk premenopausal women with breast cancer who receive adjuvant chemotherapy." N. Engl. J. Med. 337 (1997):945-955.

[5] M. Overgaard, M. Jensen, J. Overgaard, et al., "Postoperative radiotherapy in high- 
risk postmenopausal breast-cancer patients given adjuvant tamoxifen: Danish Breast Cancer Cooperative Group DBCG 82c randomised trial." The Lancet 353 (1999):16411648.

[6] T.A. Buchholz, W.A. Woodward, Z. Duan, et al., "Radiation use and long-term survival in breast cancer patients with $\mathrm{T} 1, \mathrm{~T} 2$ primary tumors and one to three positive axillary lymph nodes." Int. J. Radiation Oncology Biol. Phys. 71 (2008):1022-1027.

[7] ICRP, ICRP Publication 60: 1990 Recommendations of the internal commission on radiological protection. (International Commission on Radiological Protection, Stockholm, Sweden, 1990)

[8] R. Roychoudhuri, D. Robinson, V. Putcha, J. Cuzick, S. Darby, and H. Møller, "Increased cardiovascular mortality more than fifteen years after radiotherapy for breast cancer: a population-based study.” BMC Cancer 7 (2007):9.

[9] T.R. Mackie, J. Balog, K. Ruchala, et al., "Tomotherapy." Seminars in Radiation Oncology 9 (1999):108-117.

[10] A. Ahnesjö, "Collapsed cone convolution of radiant energy for photon dose calculation in hetrogeneous media." Med. Phys. 16 (1988):577-592.

[11] A.O. Jones and I.A. Das. "Comparison of inhomogeneity correction algorithms in small photon fields." Med. Phys. 32 (2005):766-776. 
[12] T.A.Buchholz, et al., "Dosimetric analysis of intact breast irradiation in off-axis planes." Int. J. Radiation Oncology Biol. Phys. 39(1997):261-267.

[13] G.M. Freedman, M.L. Fowble, M. Micolaou, E.R. Sigurdson, M.H. Torosian, M.C. Boraas, and J.P. Hoffman, "Should internal mammary lymph nodes in breast cancer be a target for the radiation oncologist?" Int. J. Radiation Oncology Biol. Phys. 46 (2000):805-814.

[14] B. Fowble, A. Hanlon, G. Freedman, et al., "Internal mammary node irradiation neither decreases distant metstases nor improves survival in stage I and II breast cancer." Int. J. Radiation Oncology Biol. Phys. 47 (2000):883-894.

[15] B.C. John Cho, et al. "Intensity modulated vs. non-intensity modulated radiotherapy in the treatment of the left breast and upper internal mammary lymph node chain: a comparative planning study." Radiotherapy and Oncology 62 (2002):127-136.

[16] K.S. Smith, J.P. Gibbons, B.J. Gerbi, K.R. Hogstrom, "Measurement of superficial dose from a static tomotherapy beam." Med. Phys. 35 (2008): 769-774.

[17] A. Harsolia, L. Kestin, I. Grills, et al., "Intensity-modulated radiotherapy results in significant decrease in clinical toxicities compared with conventional wedge-based breast radiotherapy.” Int. J. Radiation Oncology Biol. Phys. 68 (2007):1375-1380.

[18] S.J. Thomas and A.C.F. Hoole. "The effect of optimization on surface dose in intensity modulated radiotherapy (IMRT)." Phys. Med. Biol. 49 (2004):4919-4928. 
[19] ICRU, ICRU Report 50. Prescribing, Recording and Reporting Photon Beam Therapy (International Commission on Radiation Units and Measurements, Bethesda, MD, 1993).

[20] ICRU, ICRU Report 62. Prescribing, Recording and Reporting Photon Beam Therapy (Supplement to ICRU Report 50) (International Commission on Raditation Units and Measurements, Bethesda, MD, 1999).

[21] N.J. Yue, X. Li, S. Beriwal, D.E. Heron, M.R. Sontag, and M.S. Huq. "The intrafraction motion induced dosimetric impacts in breast 3D radiation treatment: A 4DCT based study." Med. Phys. 34 (2007):2789-2800.

[22] R. George, P.J. Keall, V.R. Kini, S.S. Vedam, J.V. Siebers, Q. Wu, M.H. Lauterbach, D.W. Arthur, and R. Mohan. "Quantifying the effect of intrafraction motion during breast IMRT planning and dose delivery.” Med. Phys. 30 (2003):552-562.

[23] R.P. Smith, P. Bloch, E.E. Harris, et al., "Analysis of interfraction and intrafraction variation during tangential breast irradiation with an electronic portal imaging device." Int. J. Radiation Oncology Biol. Phys. 62 (2005):373-378.

[24] M. Ding, J. Li, J. Deng, E. Fourkal, and C.-M. Ma, "Dose correlation for thoracic motion in radiation therapy of breast cancer." Med. Phys. 30 (2003):2520-2529.

[25] AAPM, Report of AAPM Task Group 76. The Management of Respiratory Motion in 
Radiation Oncology. (American Association of Physicists in Medicine, College Park, $\mathrm{MD}, 2006)$.

[26] K.E. Sixel, M.C. Aznar, Y.C. Ung, "Deep inspiration breath hold to reduce irradiated heart volume in breast cancer patients." Int. J. Radiation Oncology Biol. Phys. 49 (2001):199-204.

[27] G. Gagliardi, I. Lax, S. Söderdtröm, G. Gyenes, L.E. Rutqvist, "Prediction of excess risk of long-term cardiac mortality after radiotherapy of stage I breast cancer." Radiotherapy and Oncology 46 (1998):63-71.

[28] J.H. Borger, M.J. Hooning, L.J. Boersma, et al., "Cardiotoxic effects of tangential breast irradiation in early breast cancer patients: the role of irradiated heart volume." Int. J. Radiation Oncology Biol. Phys. 69 (2007):1131-1138.

[29] J. Ragaz, S.M. Jackson, N. Le, et al., "Adjuvant radiotherapy and chemotherapy in node-positive premenopausal women with breast cancer." N. Engl. J. Med. 337 (1997):956-962.

[30] S.S. Vedam, P.J. Keall, V.R. Kini, et al., "Acquiring a four-dimensional computed tomography dataset using an external respiratory signal.” Phys. Med. Biol. 48 (2003):4562.

[31] M. van Herk, P. Remeijer, C. Rasch, and J.V. Lebesque, "The probability of correct 
target dosage: dose-population histograms for deriving treatment margins in radiotherapy." Int. J. Radiation Oncology Biol. Phys. 47 (2000):1121-1135.

[32] D.M Drabik, M.A. MacKenzie, and G.B. Fallone, "Quantifying appropriate setup margins: analysis of patient setup fidelity and intrafraction motion using post-treatment megavoltage computed tomography scans.” Int. J. Radiation Oncology Biol. Phys. 68 (2007):1222-1228.

[33] E.A. White, J. Cho, K.A. Vallis et al., "Cone beam computed tomography guidance for setup of patients receiving accelerated partial breast irradiation." Int. J. Radiation Oncology Biol. Phys. 68 (2007):547-554.

[34] C.R. Ramsey, R.M. Seibert, B. Robison, and M. Mitchell, "Helical tomotherapy superficial dose measurements." Med. Phys. 34 (2007):3286-3293.

[35] A. Cherpak, R.C.N. Studinski, J.E. Cygler, "MOSFET detectors in quality assurance of tomotherapy treatments." Radiotherapy and Oncology 86 (2008):242-250.

[36] I.J. Chetty and P.M. Charland, "Investigation of Kodak extended dose range (EDR) film for megavoltage photon beam dosimetry." Phys. Med. Biol. 47 (2002):3629-3641.

[37] N.L. Childress, M. Salehpour, L. Don, et al., "Dosimetric accuracy of Kodak EDR2 film for IMRT verifications." Med. Phys. 32 (2005):539-548.

[38] Y. Yan, N. Papanikolaou, X. Weng, et al., "Fast radiographic film calibration proce- 
dure for helical tomotherapy intensity modulated radiation therapy dose verification." Med. Phys. 32 (2005):1566-1570.

[39] J.D. Fenwick, W.A. Tomé, H.A. Jaradat, et al., "Quality assurance of a helical tomotherapy machine.” Phys. Med. Biol. 49 (2004):2933-2953.

[40] N.L. Childress, I.I. Rosen. "Effect of processing time delay on the dose response of Kodak EDR2 film." Med. Phys. 31 (2004):2284-2288.

[41] A. Sankar, K.M. Ayyangar, R.M. Nehru, et al., "Comparison of Kodak EDR2 and Gafchromic EBT film for intensity-modulated radiation therapy dose distribution verification." Medical Dosimetry 31 (2006):273-282.

[42] M.W. Kissick, J. Fenwick, J.A. James et al., "The helical tomotherapy thread effect." Med. Phys. 32 (2005):1414-1423. 